\title{
Hollow cylinder tests on Boom Clay: modelling of strain localization in the anisotropic Excavation Damaged Zone
}

\author{
Bertrand François ${ }^{\mathrm{a}}$, Vincent Labiouse ${ }^{\mathrm{b}}$, Arnaud Dizier ${ }^{\mathrm{c}}$, \\ Ferdinando Marinelli ${ }^{\mathrm{d}}$, Robert Charlier ${ }^{\mathrm{e}}$, Frédéric Collin ${ }^{\mathrm{e}}$ \\ a Building, Architecture \& Town Planning Department (BATir), Université Libre \\ de Bruxelles, ULB, Belgium \\ $b$ Rock Mechanics Laboratory, Ecole Polytechnique Fédérale de Lausanne, \\ EPFL, Station 18, CH-1015 Lausanne, Switzerland \\ c EIG EURIDICE, Boeretang, 200, 2400 Mol, Belgium \\ d Laboratoire Sols Solides Structures - Risques, UJF, INPG, CNRS UMR 5521, \\ Domaine Universitaire, B.P. 53, 38041 Grenoble cedex 9, France \\ e Department ArGEnCo, Université de Liège, Chemin des Chevreuils 1, 4000 \\ Liège 1, Belgium
}

This is the accepted author's version of an article protected by copyright.

The rights are held by Springer-Verlag Wien.

The final publication is available at http://link.springer.com.

http://www.springerlink.com/openurl.asp?genre=article\&id=doi:10.1007/s00603-012-0348-5

Article published in:

Rock Mechanics and Rock Engineering (ISSN 0723-2632)

Special issue: Thermo-hydro-mechanical effects in clay host rocks

edited by Jean Sulem and Xiang Ling Li

Digital Object Identifier (DOI): DOI 10.1007/s00603-012-0348-5 


\title{
Hollow cylinder tests on Boom Clay: modelling of strain localization in the anisotropic Excavation Damaged Zone
}

\author{
Bertrand François a, Vincent Labiouse ${ }^{b}$, Arnaud Dizier c, Ferdinando \\ Marinelli $^{\mathrm{d}}$, Robert Charlier ${ }^{\mathrm{e}}$, Frédéric Collin ${ }^{\mathrm{e}}$. \\ ${ }^{a}$ Building, Architecture \& Town Planning Department (BATir), Université Libre de Bruxelles, ULB, Belgium \\ ${ }^{b}$ Rock Mechanics Laboratory, Ecole Polytechnique Fédérale de Lausanne, EPFL, Station 18, CH - 1015 \\ Lausanne, Switzerland \\ ${ }^{c}$ EIG EURIDICE, Boeretang, 200, $2400 \mathrm{Mol}$, Belgium \\ ${ }^{d}$ Laboratoire Sols Solides Structures - Risques, UJF, INPG, CNRS UMR 5521, Domaine Universitaire, B.P. 53 , \\ 38041 Grenoble cedex 9, France \\ ${ }^{e}$ Department ArGEnCo, Université de Liège, Chemin des Chevreuils 1, 4000 Liège 1, Belgium \\ Corresponding author: E-mail: bertrand.francois@ulb.ac.be
}

\section{$\underline{\text { ABSTRACT }}$}

Boom Clay is extensively studied as a potential candidate to host underground nuclear waste disposal in Belgium. To guarantee the safety of such a disposal, the mechanical behaviour of the clay during gallery excavation must be properly predicted. In that purpose, a hollow cylinder experiment on Boom Clay has been designed to reproduce, in a small-scale test, the Excavation Damaged Zone (EDZ) as experienced during the excavation of a disposal gallery in the underground. In this paper, the focus is made on the hydro-mechanical constitutive interpretation of the displacement (experimentally obtained by medium resolution X-Ray tomography scanning). The coupled hydro-mechanical response of Boom Clay in this experiment is addressed through finite element computations with a constitutive model including strain hardening/softening, elastic and plastic cross-anisotropy and a regularisation method for the modelling of strain localization processes. The obtained results evidence the directional dependency of the mechanical response of the clay. The softening behaviour induces transient strain localization processes, addressed through a hydro-mechanical second grade model. The shape of the obtained damaged zone is clearly affected by the anisotropy of the materials, evidencing an eye-shaped excavation damaged zone. The modelling results agree with experiments not only qualitatively (in terms of the shape of the induced damaged zone) but also quantitatively (for the obtained displacement in three particular radial directions).

\section{KEYWORDS}

Numerical modelling, anisotropy, strain localization, Boom Clay, hollow cylinder test. 


\section{Introduction}

In the coming years, safe and definitive solutions will likely be completed for managing the large quantities of high-level radioactive wastes that stem mainly from nuclear electricity production. Deep geological repositories constitute one of the most promising options to isolate such wastes from the human environment. To prevent a potential failure of these confining structures, analysis and predictions about the long-term behaviour of such disposal installations and their surrounding barriers should be based on robust science (Chapman and McKinley 1987). However, since this matter is urgent, yet concerns long time periods, the safety assessment of this option must be based mainly on predictions. Powerful simulation tools, such as finite element codes, are of paramount importance to realize this goal. Indeed, advanced numerical models have been developed and may allow these tools to reproduce, with sufficient confidence, the response of the involved geological materials subjected to the complex loadings which are characteristic of such repositories (Gens and Olivella 2001; Laloui et al. 2008).

The perturbation of the geological system caused by the gallery excavation introduces various highly coupled hydro-mechanical processes. In particular, the tunnel construction leads to significant changes in the local stress field. The stresses normal to the interface (e.g., radial stresses) are considerably reduced at the interface and may even vanish in absence of lining, while orthoradial stresses may increase significantly. It may lead to local failure under extension and/or shear. Such processes must be considered in interaction with the dissipation of pore water pressure induced by the draining gallery. Hard clays tend to show bedding plane splitting and buckling around the gallery while in more plastic clays (like in Boom Clay, the formation studied in this work), the observed failure mechanisms around the tunnel consist essentially in pure shear failure, leading to conjugated shear fractures (Blümling et al. 2007; Labiouse and Vietor, 2013). The zone in which major hydro-mechanical modifications occur is called the Excavation Damaged Zone (EDZ) (Davies and Bernier 2005; Tsang et al. 2005). 
To ensure their efficiency, the models that are expected to be used to predict and characterize the EDZ should be validated under characteristic loadings similar to the ones occurring during gallery excavations. To achieve this challenging task, simulations reproducing analogue studies have to be performed. In that context, the TIMODAZ project, a four-year specific targeted project (2006-2010), has been co-funded by the European Commission to investigate the thermal impact on the damaged zone around a radioactive waste disposal in clay host rocks. The modelling work together with the results of the lab and the in-situ tests aim at giving clear indication on the evolution of the EDZ with time and with temperature evolution. In this paper, the focus is made on the excavation-induced damage before the radioactive waste heat emission. Non-conventional hollow cylinder tests have been designed and performed on Boom Clay at the LMR-EPFL laboratory (Labiouse et al. 2013). It consists in hollow cylinder samples of Boom Clay which are submitted to mechanical loadings fairly similar, but at small-scale, to the evolution that will be encountered during gallery excavation. In parallel to the experimental aspects, focus has been made on numerical simulations in order, first, to predict the expected behaviour of Boom Clay in the experiment and, then, to interpret the obtained results in terms of constitutive behavioural features.

The Boom Clay is an overconsolidated plastic clay that is being considered as a candidate host rock for the Belgian disposal of radioactive waste. Many of its properties are favourable to the choice of this formation to fill the function of geological barrier for radioactive waste disposal (Barnichon and Volckaert 2003; Bernier et al. 2007). In the hollow cylinder experiment, due to the unloading of the central hole, the Boom Clay undergoes large strains and displacements along with a strain softening process. In such a case, localized ruptures (i.e., shear bands) may occur (Desrues and Viggiani 2004). This phenomenon has been extensively studied from a theoretical and numerical point of view and it has been proved that an enhanced model incorporating an internal length is necessary to provide an objective description of post localized loading paths (Vardoulakis and Sulem 1995). In this paper, a coupled local second gradient model (Collin et al. 2006) is used to study the possible strain localization of the Boom Clay in a coupled hydro-mechanical context. 
In the past, the issue of strain localization in hollow cylinder tests has already been addressed by numerical simulations incorporating strain softening and regularization technique to properly reproduce failure due to progressive localisation of deformation (Papanastasiou and Vardoulakis 1989; Zervos et al., 2001; Papanastasiou et al., 2008). Regarding to those significant contributions, the present simulations extend the study to hydro-mechanical conditions, including the consolidation process around the hole. It leads to a transient response of the system. Also, through the calibration of the model parameters, including elasto-plastic anisotropy, it is demonstrated that the numerical model is able to fit with experimental results.

In the framework of the hollow cylinder experiment, three main features of its behaviour have been particularly studied: the strain softening behaviour, the anisotropic mechanical response, and the localization of deformations. In order to model the mechanical cross-anisotropy and the strain localization, the problem has been treated in two-dimensional plane strain conditions. It leads to a proper representation of the non-axisymmetric and time-dependent damaged zone. The mechanical behaviour of the clay has been modelled by a hardening/softening Drucker-Prager model including elastic and plastic cross-anisotropy.

This work contributes to the understanding of the irreversible processes occurring in the clay host formations due to excavation and drainage phases. Firstly, the characteristics of the hollow cylinder experiment, the theoretical formulation of the constitutive and numerical tools are presented. Secondly, the experiment is simulated and the results are compared with the laboratory measurements in terms of displacement. Finally, those results are interpreted in the light of the hydro-mechanical couplings, the elasto-plastic response, the strain localization and the mechanical anisotropy.

\section{Material and experiment characteristics}

\subsection{Boom Clay}

At Mol-Dessel nuclear site, the Boom Clay is present 190-290 m below ground level and is surrounded by water-bearing sand layers, above and below it. The Boom Clay behaviour is 
characterised by a highly non-linear stress-strain response. The overconsolidation ratio (OCR) is about 2.4. The total vertical stress and pore water pressure at the level of the Underground Research Laboratory HADES are respectively some 4.5 and 2.2 MPa (Bernier et al. 2007). Table 1 summarizes the main geomechanical parameters of Boom Clay, determined by back-analysis of measurements done during galleries excavations. Those parameters consist in a first approximation of the behaviour of Boom Clay that encounters more complex phenomena such as mechanical anisotropy and hardening/softening processes. One of the key issues of the present work has been to evaluate the role of those aspects on the global response of a gallery excavated in Boom Clay.

\section{Table 1}

\subsection{Hollow cylinder test}

Laboratory experiments carried out at the LMR-EPFL on thick-walled hollow cylindrical samples aim at reproducing, at small-scale, conditions similar to those that will be experienced by host rocks around disposal galleries for heat emitting radioactive waste. The test that has been numerically simulated in this paper has been performed on a Boom Clay sample cored parallel to the bedding planes and subjected to a decrease of the confining pressure in the central hole of the hollow cylinder. The hollow cylindrical samples were prepared by drilling a coaxial central hole in a drill core (14 and $86 \mathrm{~mm}$ in diameter respectively). The preparation of the sample was carefully performed in order to minimize the sample disturbance. No signs of prior-to-testing or preparation-induced damage were detected visually and in medium resolution XRCT scans. To complete the tests, the outer and inner confining pressures as well as the outer and inner drain pressures were controlled separately. A sketch of the experimental set-up is shown in Figure 1. After recovery of the in-situ stress conditions encountered in Boom Clay at $223 \mathrm{~m}$ depth (isotropic total stress field of 4.5 MPa and pore water pressure of $2.2 \mathrm{MPa}$ ), the pressure in the central hole was reduced to model a gallery construction and to induce a damaged zone, similarly to the Excavation Damaged Zone observed around galleries in the HADES laboratory. 
In terms of mechanical conditions, the inner and outer boundaries of the hollow cylinder were stress controlled. The confining pressure at the external boundary was kept constant at $4.5 \mathrm{MPa}$ all along the test, while the internal confining pressure is decreased down to $1.0 \mathrm{MPa}$ in 70 minutes. In terms of hydraulic conditions, a constant pore water pressure of 2.2 MPa was imposed at the outer boundary while the water pressure in the inner drain decreased from 2.2 MPa to 0.6 MPa in 70 minutes. The pressure boundary conditions at the end of the unloading were then kept constant to allow pore water pressure redistribution inside the sample.

\section{Figure 1}

For more completeness, the testing conditions and the obtained experimental results are fully detailed in Labiouse et al. (2013). Among the various tests that have been performed on Boom Clay (test 13A, 13B-Bis and 14-Bis), the results of the test 13A have been considered for the present numerical simulations.

To study the development and the evolution of the damaged zone induced around the central hole without removing the sample from the testing cell, medium resolution X-Ray Computed Tomography (XRCT) scans were carried out at different steps of the experiments. The comparison of the mid-plane cross-sections obtained by the tomography scans before and after the hydro-mechanical unloading clearly evidences four essential features of behaviour (Figure 2).

(i) The mechanical irreversibility: The closure of the hole is such that the produced strains are mainly plastic.

(ii) The dilatancy/softening response: The cross-sectional area is larger after unloading than before which is characteristics of dilatancy upon shearing that usually occurs together with a softening process.

(iii) The mechanical anisotropic response: During closure, the circular hole is oval-shaped because of the directional dependency of the mechanical behaviour. 
(iv) The strain localization: the non-regular shape of the hole after closure is an indication of some localizations of the deformation.

The dimension of the oval-shaped central hole is reported in Figure 3 showing the radius of the deformed hole as a function of the orientation with respect to the bedding direction. The displacement in the direction parallel to bedding is more than twice larger than the displacement perpendicularly to bedding. Consequently, the measured displacements have been analysed in three particular directions: in the bedding direction $\left(\theta=0^{\circ}\right)$, with an angle of $45^{\circ}\left(\theta=45^{\circ}\right)$ with respect to the bedding planes and perpendicular to bedding $\left(\theta=90^{\circ}\right)$.In addition, it has been also possible to evaluate the displacement of pyrite inclusions present in the specimens by tracking their movement between the scans before and after the mechanical unloading. The measured displacement profiles for $\theta=0^{\circ}, 45^{\circ}$ and $90^{\circ}$ are reported in Figure 4. They will be compared with the numerical predictions further in the paper (Figures 9 and 12).

\section{Figure 2}

\section{Figure 3}

\section{Figure 4}

\section{Macroscopic balance equations}

The hydro-mechanical processes governing the phenomena are studied by the finite element method (Collin et al. 2002). The field equations consider the medium as a deformable two-phase material (i.e., a water saturated medium) in which mass transfers occur. In this section, field equations implemented in the LAGAMINE finite element code (Charlier et al. 2001; Collin 2003), which has been used for the computations, are presented.

With water saturated conditions being assumed, the balance equations are obtained by considering that the soil is composed of solid matrix and voids fully filled with water. The equilibrium and balance equations, as well as the water flow, are expressed in the moving current configuration through a 
Lagrangian actualised formulation (Charlier 1987). According to these assumptions, there is no evolution of solid skeleton mass and the mass balance equation of the solid skeleton is necessarily met. For a given mixture volume, the mass balance equation reads:

$$
\frac{\partial \rho_{s}(1-n)}{\partial t}=0
$$

where $\rho_{s}$ is the density of solid grains, $n$ the soil porosity and $t$ the time.

The mass conservation equation for the water is:

$$
\frac{\partial \rho_{w} n}{\partial t}+\operatorname{div}\left(\rho_{w} \mathbf{f}_{\mathbf{w}}\right)=0
$$

where $\boldsymbol{f}_{w}$ is the macroscopic velocity of the water with respect to the solid phase. $\rho_{w}$ is the liquid water density

The liquid water motion is governed by the Darcy's law:

$$
\mathbf{f}_{\mathbf{w}}=-\frac{k_{w}}{\mu_{w}}\left(\operatorname{grad}\left(p_{w}\right)\right)
$$

where $k_{w}$ is the intrinsic water permeability, assumed to be isotropic, $\mu_{w}$ the dynamic viscosity of the water.

The soil equilibrium equation is given by:

$$
\operatorname{div}\left(\sigma_{i j}\right)=0
$$


where $\sigma_{i j}$ is the total (Cauchy) stress tensor, with compressive stress taken as positive. The behaviour of the solid matrix is assumed to be governed by the Terzaghi's effective stress tensor $\sigma_{i j}^{\prime}$ :

$\sigma_{i j}^{\prime}=\sigma_{i j}-p_{w} \delta_{i j}$

where $\delta_{i j}$ is the Kronecker's symbol.

In this Lagrangian approach, the Cauchy strain tensor is used:

$\varepsilon_{i j}=\frac{1}{2}\left(L_{i j}-L_{j i}\right)$

where $L_{i j}=\frac{\partial u_{i}}{\partial X_{j}}$ is the displacement $\left(u_{i}\right)$ gradient defined in global axis $\left(X_{j}\right)$ in the moving current configuration (Charlier et al. 2001). This strain tensor is related to the Terzaghi's effective stress tensor through the constitutive model addressed in the next section.

Thereby, Equations (2) and (4) compose the field equations with the three degrees of freedom as unknowns (pore water pressure and the two solid displacements).

\section{Constitutive models and parameters}

\subsection{Elasto-plasticity for cross-anisotropic material}

In addition to the conventional elasto-plastic behaviour of Boom Clay, the main objective of the numerical simulations was to improve our understanding on some specificities of the Boom Clay rheology. In particular, aspects on the mechanical anisotropy and the softening processes have been investigated. To that end, a hardening/softening Drucker-Prager model (a frictional elasto-plastic 
model) considering elastic and plastic cross-anisotropy has been developed and implemented in the LAGAMINE finite element code.

The model uses elastic cross-anisotropy coupled with an extended Drucker-Prager hardening/softening plasticity model. The plastic cross-anisotropy is taken into account through the material cohesion that depends on the angle between major principal stress and the normal to the bedding orientation. The behaviour is isotropic in the plane of bedding and the unique direction of anisotropy is perpendicular to bedding. The properties of such cross-anisotropic materials are independent of rotation about an axis of symmetry normal to the bedding plane. In such a situation, the elasto-plastic stress-strain relations are more convenient to be expressed in the anisotropic axes which are linked to the bedding orientation

The elasto-plasticity principle allows that the total strain rate, $\dot{\varepsilon}_{i j}$, be split into elastic, $\dot{\varepsilon}_{i j}^{e}$, and plastic, $\dot{\varepsilon}_{i j}^{p}$, components :

$\dot{\varepsilon}_{i j}=\dot{\varepsilon}_{i j}^{e}+\dot{\varepsilon}_{i j}^{p}$

$\dot{\varepsilon}_{i j}^{e}$ is linked to stress rate through the Hooke law :

$\dot{\varepsilon}_{i j}^{e}=D_{i j k l}^{e} \dot{\sigma}_{k l}^{\prime}$

The $D_{i j k l}^{e}$ matrix considers cross-anisotropic elasticity that requires five independent parameters, as follow (Lekhnitskii 1977; Graham and Houlsby 1982): 


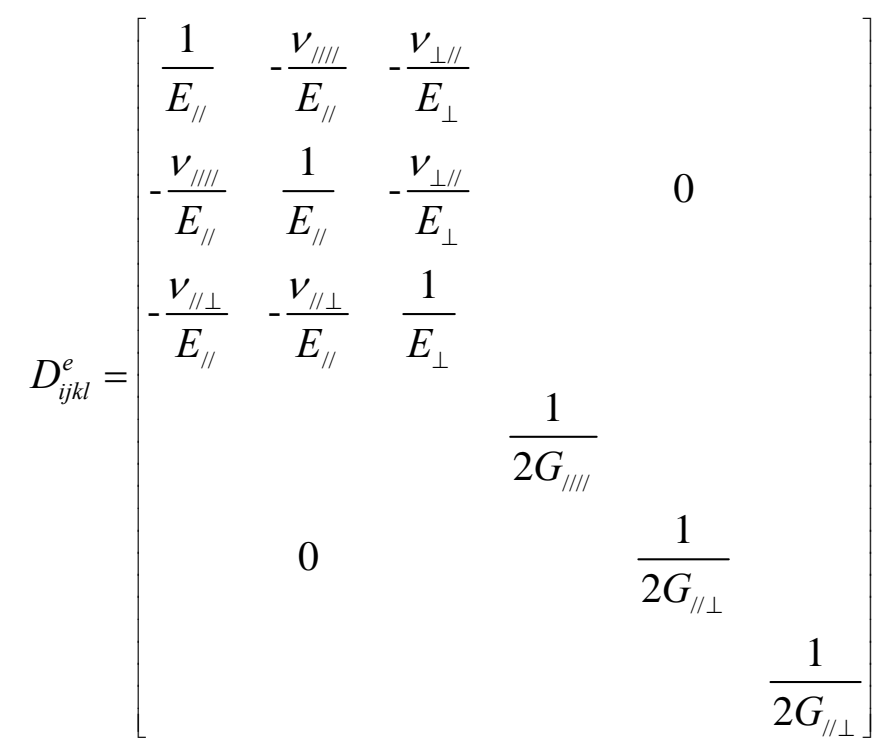

The symmetry of the stiffness matrix imposes that

$$
\frac{v_{\perp / /}}{E_{\perp}}=\frac{v_{/ / \perp}}{E_{/ /}}
$$

The Drucker-Prager plastic yield limit, flow rule and consistency condition are expressed in the anisotropic axis. This way of proceed aims at keeping the hardening matrix as simple as possible, with 18 terms of the matrix equal to zero (meaning that the i-j component of shear strain can only be produced by the i-j component of shear stress).

The limit between the elastic and the plastic domain is represented by a yield surface in the principal stress space. This surface corresponds to the Drucker-Prager yield surface $f$ (Drucker and Prager 1952) (Figure 5):

$$
f \equiv q-m\left(p^{\prime}+\frac{c^{\prime}}{\tan \phi^{\prime}}\right)=0
$$

with 
$m=\frac{6 \sin \phi^{\prime}}{3-\sin \phi^{\prime}}$

$p^{\prime}$ and $q$ are the mean effective stress and the deviatoric stress and $c^{\prime}$ and $\phi^{\prime}$ are the cohesion and the friction angle, respectively

\section{Figure 5}

In sedimentary rocks, it is generally observed that the strength of the materials depends on the loading direction with respect to the bedding plane (Duveau et al. 1998). In the present model, the material cohesion depends on the angle between major principal stress and the normal to the bedding plane (François et al. 2011). Three cohesion values are defined, for major principal stress parallel $\left(\alpha_{\sigma_{1}}=0^{\circ}\right.$ ), perpendicular $\left(\alpha_{\sigma_{1}}=90^{\circ}\right)$ and with an angle of $45^{\circ}\left(\alpha_{\sigma_{1}}=45^{\circ}\right)$ with respect to the normal to bedding plane. Between those values, cohesion varies linearly with $\alpha_{\sigma_{1}}$. The mathematical expression of the cohesion is as follows (Figure 6):

$c_{i \text { or } f}^{\prime}=\max \left[\left(\frac{c_{i \text { or } f\left(45^{\circ}\right)}^{\prime}-c_{i \text { or } f\left(0^{\circ}\right)}^{\prime}}{45^{\circ}}\right) \alpha_{\sigma_{1}}+c_{i \text { or } f\left(0^{\circ}\right)}^{\prime} ;\left(\frac{c_{i \text { or } f\left(90^{\circ}\right)^{\circ}}^{\prime}-c_{i \text { or } f\left(45^{\circ}\right)}^{\prime}}{45^{\circ}}\right)\left(\alpha_{\sigma_{1}}-45^{\circ}\right)+c_{i \text { or } f\left(45^{\circ}\right)}^{\prime}\right]$

$i$ and $f$ indexes correspond, respectively, to the initial and final cohesions of the hardening equation (19). $\alpha_{\sigma_{1}}$ is the angle between the normal to the bedding plane $\vec{n}$ and the major principal stress $\vec{\sigma}_{1}$

$\alpha_{\sigma_{1}}=\cos ^{-1}\left(\frac{\vec{n} \cdot \vec{\sigma}_{1}^{\prime}}{\|\vec{n}\|\left\|\vec{\sigma}_{1}^{\prime}\right\|}\right)$

\section{Figure 6}


A general non-associated plasticity framework is considered:

$\dot{\varepsilon}_{i j}^{p}=\lambda^{P} \frac{\partial g}{\partial \sigma_{i j}^{\prime}}$

with the plastic potential $g$ defined as:

$g \equiv q-m^{\prime} p^{\prime}$

with

$m^{\prime}=\frac{6 \sin \psi}{3-\sin \psi}$

where $\psi$ is the dilatancy angle.

The model is a hardening Drucker-Prager model that allows hardening/softening processes during plastic flow. This is introduced via an hyperbolic variation of the friction angle and the cohesion between initial $\left(\phi_{i}^{\prime}\right.$ and $\left.c_{i}^{\prime}\right)$ and final $\left(\phi_{f}^{\prime}\right.$ and $c_{f}^{\prime}$ ) values as a function of the Von Mises equivalent plastic strain $\varepsilon_{e q}^{p}$ (Barnichon 1998):

$$
\begin{gathered}
\phi^{\prime}=\phi_{i}^{\prime}+\frac{\left(\phi_{f}^{\prime}-\phi_{i}^{\prime}\right) \varepsilon_{e q}^{p}}{B_{p}+\varepsilon_{e q}^{p}} \\
c^{\prime}=c_{i}^{\prime}+\frac{\left(c_{f}^{\prime}-c_{i}^{\prime}\right) \varepsilon_{e q}^{p}}{B_{c}+\varepsilon_{e q}^{p}}
\end{gathered}
$$


where the Von Mises equivalent plastic strain $\varepsilon_{e q}^{p}$ is obtained by integration of the Von Mises equivalent plastic strain rate:

$$
\varepsilon_{e q}^{p}=\int_{0}^{t} \sqrt{\frac{2}{3}\left(\dot{\varepsilon}_{i j}^{p}-\frac{\dot{\varepsilon}_{v}^{p}}{3} \delta_{i j}\right)\left(\dot{\varepsilon}_{i j}^{p}-\frac{\dot{\varepsilon}_{v}^{p}}{3} \delta_{i j}\right)} d t
$$

$B_{p}$ and $B_{c}$ are material parameters.

\subsection{HM second grade model}

The degradation of the mechanical properties of geomaterials can be due to increasing porosity in granular materials, or by development of micro-fractures in cohesive materials. In order to model such behavior, constitutive equations involving softening are used and they usually predict failure in a localized mode. In this case, numerical modelling of localized shear zones suffers of pathological mesh-sensitivity. It has been proved that an enhanced model incorporating an internal length is necessary to provide an objective description of post localized loading paths. Many enhancements have been proposed and studied in the past, since the pioneering work of Aifantis (1984) (see Vardoulakis and Sulem (1995)). The non-local approach was first proposed for damage concrete mechanics (Bazant et al. 1984) and extended later to other materials. Another type of enhancement corresponds to constitutive models with gradient of internal variables or with non-local internal variables (de Borst and Muhlhaus 1992; Vardoulakis and Sulem 1995). A last family of enriched models comes from continua with microstructure. This most ancient one is the well-known Cosserat model (Cosserat and Cosserat 1909) (see Muhlhaus and Vardoulakis (1987) or Vardoulakis and Sulem (1995) for applications to geomaterials) but local second gradient formulation has been more recently proposed (Mindlin 1965; Mindlin and Eshel 1968; Germain 1973a; Germain 1973b) (see Chambon et al. (1998); Chambon et al. (2001); Matsushima (2002) for application to geomaterials). This latter model will be used in the following as a regularization technique for the coupled hydro-mechanical modelling. More details on this coupled second gradient model can be found in Collin et al. (2006). 
In the framework of microstructure continuum theory, a micro-kinematic gradient field $v_{i j}$ is introduced to describe strain and rotation at the microscale. With respect to classical continuum mechanics, additional terms are then added in the internal virtual work of a given body (Germain 1973a). In the local second gradient model used in the following, an assumption is added: the microkinematic gradient is equal to the macro-deformation gradient. The balance equations of the coupled second gradient model can thus be written as:

$$
\begin{aligned}
& \int_{\Omega}^{t}\left(\sigma_{i j}^{t} \frac{\partial u_{i}^{*}}{\partial x_{j}^{t}}+\Sigma_{i j k}^{t} \frac{\partial^{2} u_{i}^{*}}{\partial x_{j}^{t} \partial x_{k}^{t}}\right) d \Omega^{t}=\int_{\Gamma_{\sigma}^{t}}\left(\bar{t}_{i} u_{i}^{*}+\bar{T}_{i} D u_{i}^{*}\right) d \Gamma^{t} \\
& \int_{\Omega}^{t}\left(\dot{M}^{t} p_{w}^{*}-m_{i}^{t} \frac{\partial p_{w}^{*}}{\partial x_{i}^{t}}\right) d \Omega^{t}=\int_{\Omega^{t}}^{t} Q^{t} p_{w}^{*} d \Omega^{t}-\int_{\Gamma}^{t} \bar{q}_{q}^{t} p_{w}^{*} d \Gamma^{t}
\end{aligned}
$$

where $\Sigma_{i j k}^{t}$ is the double stress associated to the second gradient of the displacement field, $\bar{t}_{i}$ is the external (classical) forces per unit area and $\bar{T}_{i}$ an additional external (double) force per unit area, both applied on a part $\Gamma_{\sigma}^{t}$ of the boundary of $\Omega^{t}$.D denotes the normal derivative of any quantity $q$, $(D q$ $\left.=\left(\partial q / \partial x_{k}\right) n_{k}\right)$

In the governing equations (21-22), it is assumed that pore fluid has no influence at the microstructure level: micro-kinematic gradients are not generated by pore pressure variations. This latter hypothesis follows the ideas of Ehlers and Volk (1998) concerning a Cosserat model for biphasic medium. Cosserat model (Cosserat and Cosserat 1909) is in fact a particular case of medium with microstructure.

The previous virtual work equation of second gradient models can of course be used in a finite element code. However, this equation needs the use of $C^{l}$ functions for the displacement field as second derivatives of the displacement are involved. In order to avoid such functions in the virtual 
work principle (21), the equalities between micro-kinematic gradient and macro-deformation gradient are introduced through a field of Lagrange multipliers $\lambda_{i j}$ related to a weak form of the equality constraint (Matsushima et al. 2002):

$$
\begin{aligned}
& \int_{\Omega}^{t}\left(\sigma_{i j}^{t} \frac{\partial u_{i}^{*}}{\partial x_{j}^{t}}+\Sigma_{i j k}^{t} \frac{\partial v_{i j}^{*}}{\partial x_{k}^{t}}\right) d \Omega^{t}-\int_{\Omega} t \lambda_{i j}^{t}\left(\frac{\partial u_{i}^{*}}{\partial x_{j}^{t}}-v_{i j}^{*}\right) d \Omega^{t}-W_{e}^{*}=0 \\
& \int_{\Omega} t \lambda_{i j}^{*}\left(\frac{\partial u_{i}^{t}}{\partial x_{j}^{t}}-v_{i j}^{t}\right) d \Omega^{t}=0 \\
& \int_{\Omega}^{t}\left(\dot{M}^{t} p_{w}^{*}-m_{i}^{t} \frac{\partial p_{w}^{*}}{\partial x_{i}^{t}}\right) d \Omega^{t}=\int_{\Omega^{t}}^{t} Q^{t} p_{w}^{*} d \Omega^{t}-\int_{\Gamma}^{t} \bar{q}_{q}^{t} p_{w}^{*} d \Gamma^{t}
\end{aligned}
$$

In the present work, the stress tensor $\sigma_{\mathrm{ij}}$ in Equation (23) is a total stress defined according to Terzaghi's postulate (5). On the contrary, the double stress $\Sigma_{\mathrm{ijk}}$ has no link with the pore pressure. One of the advantages of local second gradient is the fact that constitutive equations remain local and therefore it is straightforward to formulate a second gradient extension of any mechanical constitutive models like the cross-anisotropic Drucker-Prager model developed in this work.

\subsection{Model parameters}

The use of the diffusive and mechanical constitutive models requires the definition of material parameters in agreement with the hydro-mechanical behaviour of the Boom Clay. The mechanical parameters have been assigned on the basis of the extensive literature and experimental studies performed in the framework of the TIMODAZ project (Timodaz 2010; Dizier 2011). However, experimental laboratory evidences on the mechanical anisotropic characters of the Boom Clay has never been properly quantified. Indeed, there are no clear homogeneous laboratory tests focusing on the dependency of mechanical parameters on the direction of loading with respect to bedding plane. 
So, starting from averaged isotropic parameters, the specific parameters of the anisotropic DruckerPrager model (i.e., the anisotropic elasticity and cohesion) have been adjusted to fit as much as possible with the experimental results of the hollow cylinder test. Let's note that the calibrated anisotropic ratio of the Young's moduli $\left(\mathrm{E}_{/ /} / \mathrm{E}_{\perp}\right)$ is in agreement with the back-analysis performed by Chen et al. (2011) in the framework of the ATLAS experiment in Boom Clay. Actually, two sets of parameters have been considered for the Drucker-Prager model. First, an isotropic behaviour has been considered as a preliminary modelling. Then, the anisotropic characteristics of the clay have been included in a second step of computation. The mechanical parameters are reported in Table 2.

The second gradient model needs some parameters associated with the relation between the double stresses and the second gradient of the displacement. A simplified linear relation has been used in the present computations following what has been done in Collin et al. (2006). This involves only one additional parameter called $\beta$ which governs the width of the localized zones. Actually, Chambon et al. (1998) showed that the internal length is proportional to the square root of the ratio $\beta$ over the constitutive modulus of the first gradient law. This latter modulus being fixed, we can adjust the parameter $\beta$ in order to control the width of the shear band. In the following computations $\beta=3$ Newton. The parameters governing the hydraulic diffusion in Boom Clay, reported in Table 3, were directly established from a literature analysis (Bernier et al. 2007; Bastiaens et al. 2006; François et al. 2009).

\section{Table 2}

Table 3

\section{$\underline{\text { 5. Numerical simulations }}$}

\subsection{Features of the analysis}

The objective of this simulation is to reproduce the behaviour of the material in the mid-plane section of the hollow cylinder, assuming plane strain conditions in the axial direction. Because of the 
consideration of mechanical cross-anisotropy, a directional dependency of the encountered behaviour is expected. Also, the possible strain localization may produce a loss of the symmetry of the system. Consequently, the problem is not axisymmetric and must be addressed through a two-dimensional plane strain mesh. A quarter of the entire section has been considered (Figure 7). The clay sample is considered as homogeneous and to be fully saturated.

Because of the problem symmetry, the radial boundaries of the quarter section are assumed impervious and their displacements are perpendicularly constrained. Second gradient models need additional boundary conditions: we can fix either the so-called external double forces or the corresponding gradient of the displacement. Along the axis of symmetry, the normal derivative of the radial displacement has been constrained, in order to enforce symmetry (Zervos et al. (2001) and Zervos et al. (2008)). On the rounded boundaries, the external double forces have been taken equal to zero in the reported computations. The mesh size has been defined in order to be able to obtain objective solutions with the chosen value of $\beta$.

The simulations have been performed until reaching a hydro-mechanical steady-state that has been obtained after $45000 \mathrm{~s}$ (12 hours 30 minutes). The entire boundary conditions are summarized in Figure 8.

\section{Figure 7}

\section{Figure 8}

The computations have been performed in two steps. First, as a preliminary step, an isotropic case has been considered. A one-dimensional axisymmetric problem has been considered. By forcing the response to be fully axisymmetric, strain localization has not been considered. Also, the elastic modulus and the cohesion have been taken constant, without directional dependency, as reported in the first column of Table 3. Consequently, the response can be studied in a single radial profile which makes the interpretation more straightforward. The objective is to address the problem in a progressive 
manner. Then, in a second step, anisotropy and strain localization have been considered inducing a clear directional dependency of the behaviour. For this second part, the results of the numerical simulations have been studied in three particular directions (parallel to bedding, at $45^{\circ}$ with respect to bedding and perpendicular to bedding). The results of the two cases are successively addressed in the two next sections.

\subsection{Isotropic approximation}

The objective of this first isotropic case is to understand the hydro-mechanical couplings together with the softening/hardening response of the material on a simple case (leaving aside the directional dependency). Figure 9 exhibits the radial profile of the displacements. The isotropic Drucker-Prager model predicts $2.6 \mathrm{~mm}$ and $0.4 \mathrm{~mm}$ displacements of the inner and outer faces, respectively. The shape and the order of magnitude of the displacement profiles predicted by the isotropic Drucker-Prager model are in good agreement with the experimental measurements. This is particularly noticeable, knowing that the material parameters of both models have been determined from an independent literature review. Indeed, the obtained results consist in blind predictions performed before the carrying out of the experiment. However, of course, the directional dependency of the results is not reproduced because of the assumption of this preliminary simulation (axisymmetric conditions, no anisotropy and no localisation of deformation).

\section{Figure 9}

In terms of pore water pressure evolution, the effects of the mechanical unloading and the drainage of the hole are twofold (Figure 10a). In the internal part of the cylinder, the pore water pressure dissipates following the drop of water pressure in the inner drain. On the contrary, in the external part of the cylinder, at short term $(t=4200 \mathrm{~s})$, the pore water pressure increases due to a hardening process which tends to diminish the pore space. Then, during the stabilization period, the pore water pressure reaches equilibrium between the two imposed values at the inner and outer faces. The profile of the pore water 
pressure at the steady-state is independent of the mechanical and hydraulic parameters, assuming that the Boom Clay characteristics remain homogeneous.

The dilatancy angle being assumed equal to zero, the model predicts that no volumetric plastic strains are produced during plastic shearing. So, the porosity variation is only produced by the elastic component of the deformation (Figure 10b). From the initial porosity of 0.39 , the clay contracts in the external part of the cylinder and swells in the vicinity of the hole. This is consistent with the shortterm evolution of the pore water pressure (drop of pressure in the inner part and rise of pressure in the outer part).

After mechanical unloading and pore water pressure stabilisation, the profile of the radial effective stress is quasi linear between the two imposed values at the inner and outer faces. On the contrary, the orthoradial and axial effective stresses exhibit a non-linear profile (Figure 10c). Orthoradial stresses increase in the outer part because of stress redistribution around the plastic zone. It produces a hardening process in the outer part (with an increase of orthoradial and axial stresses) while the inner zone is subject to softening.

Actually, the plastic process progressively reduces from the inner to the outer regions. The evolution of the deviatoric plastic strain predicted by the isotropic model shows that the external zone is almost unaffected by plastic processes while the deviatoric plastic strain drastically increases in the $5 \mathrm{~mm}$ zone around the hole (Figure 10d).

\section{Figure 10}

Figure 11 depicts the followed stress paths in the $\left(p^{\prime}-q\right)$ plane at three locations in the hollow cylinder: at the inner face $(x=7 \mathrm{~mm})$, in the middle of the cylinder $(x=25 \mathrm{~mm})$ and at the outer face $(\mathrm{x}=43 \mathrm{~mm})$. The plotted points on the curves correspond to the stress state at the end of the 
mechanical unloading and water pressure reduction in the central hole, i.e. after $4200 \mathrm{~s}=70$ minutes. At the inner face, the process occurs under drained condition and the stress path in the $\left(p^{\prime}-q\right)$ plane is linear as long as the stress point remains in the elastic domain. Then, at the inner face, when plasticity occurs, the increment of deviatoric stress is reduced; the deviatoric stress passes through a peak value and finally decreases. This process happens along with a strong reduction of the mean effective stress during the mechanical unloading. On the contrary, on the external face, the mechanical unloading of the central hole produces a monotonic increase of both mean effective stress and deviatoric stress. This is a consequence of the increase of the orthoradial stress in the outer part because of stress redistribution around the softened zone. So, the stress state in the hollow cylinder progressively moves from a mean effective stress decrease in the inner region to a mean effective stress increase in the external zone. After the imposed reduction in pressure in the central hole (part of the stress path curves after the points plotted in Figure 11), the stress state is still evolving in the cylinder due to the on-going pore pressure redistribution.

\section{Figure 11}

\subsection{Anisotropic behaviour and strain localization}

Using the anisotropic Drucker-Prager model, the philosophy of the simulation has been to adapt material parameters characterizing the anisotropic behaviour (i.e. anisotropy of the cohesion and elastic tensor) to get the best agreement with experiment. The simulations are no more blind predictions but rather fitting and calibration. Let's note all the other parameters have been retrieved from the isotropic Drucker-Prager model, without any adjustments. This anisotropic model allows us to better reproduce the magnitude of displacements as measured experimentally, including their directional dependency. Also, in this case, the strain localization process has been considered through the use of the second grade model. 
So, the numerical results obtained with those new hypotheses exhibit quite a different pattern. The results are no more axisymmetric. The directional dependency is induced by two distinct phenomena. Firstly, cross-anisotropic elasticity together with the material cohesion that depends on the angle between major principal stress and the normal to the bedding planes make the mechanical response intrinsically anisotropic. Secondly, strain localization occurs essentially due to the strain softening cohesion characteristic of the post-peak response of the material. As a consequence in addition to the intrinsic anisotropic response, the non-symmetric orientation of the shear bands induces a second source of anisotropy. This effect is not intrinsic but depends on the evolution of the boundary conditions and the kinematics of the hydro-mechanical response of the clay.

Figure 12 compares the obtained displacement with the experimental measurement in the three considered radial sections $\left(\theta=0^{\circ}, 45^{\circ}\right.$ and $\left.90^{\circ}\right)$. The larger displacements are obtained in the direction parallel to bedding. The major principal stress being orthoradial, $c\left(0^{\circ}\right)$ corresponds to the cohesion in the direction for which the orthoradial stress is normal to the bedding planes, i.e. in the direction of bedding $\left(\theta=0^{\circ}\right)$. This direction is weaker than the perpendicular direction $\left(\theta=90^{\circ}\right)$, governed by $c\left(90^{\circ}\right)$. So, the plastic strain is larger for $\theta=0^{\circ}$, inducing larger displacements for $\theta=0^{\circ}$ than for $\theta=$ $90^{\circ}$. In the outer zone, where the plastic component of the strain is negligible, the response is mainly governed by the elastic behaviour. In that zone, the redistribution of the stress around the anisotropic damaged zone makes the displacements of the outer face larger in the direction perpendicular to bedding. The model catches well the magnitude of displacement.

\section{Figure 12}

The displacement field is mainly governed by the deviatoric strain developed in the plastic zones, i.e. in the vicinity of the hole and in the shear bands. That plastic mobilization depends on the stress state (affected by the elastic anisotropy) and on the plastic yield limit (affected by the anisotropic cohesion). As a consequence, the extension of the zone in which significant plasticity occurs is larger for $\theta=0^{\circ}$ 
(parallel to bedding) while the magnitude of plasticity is higher for $\theta$ around $45^{\circ}$. The maximum strength occurs in the direction perpendicular to bedding (for $\theta=90^{\circ}$ ) where the principal stress (i.e. the orthoradial stress), is parallel to bedding. The produced deviatoric strain is less significant in that direction. Figure 13 shows the radial profile of the deviatoric strain for $\theta=0^{\circ}, 45^{\circ}$ and $90^{\circ}$ for different times after unloading. At the end and right after the excavation (up to $t=6000 \mathrm{~s}$ ), a shear bands pattern develops progressively and the direction $\theta=0^{\circ}$ crosses two distinct shear bands that are marked by the two bumps in the deviatoric strain profile. Then, this complex pattern of shear bands does not evolve much and the shear strain increments progressively become negligible.

The final profile of deviatoric strain is the integral on time of each profile of the increment of deviatoric strain. So, the total deviatoric strain considers the history of the shear band location and its intensity. In the direction $\theta=90^{\circ}$, the strain localization occurs in the $5 \mathrm{~mm}$ zone close to the hole. The maximum deviatoric strain takes place at the inner face and it decreases rapidly with the distance to the hole. For directions tending towards $\theta=0^{\circ}$, the extension of the zone, in which significant plasticity occurs, increases. The most significant plastic strains develop on an area inside radii of 13 $\mathrm{mm}$ for $\theta=90^{\circ}$ and $27 \mathrm{~mm}$ for $\theta=0^{\circ}$, respectively.

\section{Figure 13}

The spatial and temporal distribution of the active shear bands is more noticeable on Figure 14 that shows the maps of the plastic points and the contour of the normalized increment of the deviatoric strain for different time steps. After the excavation, almost all the section is plastic in the sense that the plastic mechanism is activated considering an initial friction angle of $5^{\circ}$. However, even if most of the zone is plastic, it is clear that a shear bands pattern appears. Between these bands, the behaviour is elastic and the contour of the increment deviatoric strain highlights the activity of the bands in terms of shear strains. At $t=5000 \mathrm{~s}$ and $t=6000 \mathrm{~s}$, the process of shear banding shows that some bands develop and then become less active. The competition between these different bands is clearly 
observed. At $t=10000 \mathrm{~s}$, the localization pattern becomes more stable. It is to be emphasised that the most active shear bands occur at the beginning of the modelling (at the end and right after the excavation).

\section{Figure 14}

It is worthwhile to note that, based on the medium resolution X-Ray Tomography scan performed after reaching the steady state, there is no clear experimental evidence on the development of shear bands in the hollow cylinder. On the other hand, heterogeneities observed in the deformation of the central hole could indicate possible strain localization phenomena. In the direction of bedding, the limited strength of the clay combined with the high elastic stiffness makes larger the zone in which plastic strain accumulates. It produces an eye-shaped damage zone. It has been possible to exhibit this response in the experiment through digital image correlation (DIC) analysis. In the numerical simulations, the zone in which large shear strains occur (the so-called excavation damaged zone) compares rather well with the experiments, as shown in Figure 15.

\section{Figure 15}

There remains the question of the transferability of the experimental observations to the EDZ around real scale galleries. On one hand, the laboratory data are consistent with observations made during the construction of the connecting and Praclay galleries at Mol URL (Labiouse et al. 2013). On the other hand, in a problem governed by softening and strain localization, a scale effect is expected. Indeed, Zervos et al. (2001) showed numerically that when localization occurs small holes are stronger than larger ones (see also Papamichos and van den Hoek (1995)). Nevertheless, the present simulations show that the numerical tools are able to reproduce, qualitatively and quantitatively, the observed results around hole at the laboratory scale. The results obtained at small-scale could be easily extended to real situations by modelling a full-scale problem. However, this is out of the scope of the present study. 


\section{Conclusions}

Gallery excavation in anisotropic saturated plastic clays, such as the Boom Clay, induces highly complex phenomena (including hydro-mechanical coupling, plastic strain, softening and strain localization) in the vicinity of the gallery. The zone around the gallery in which major hydromechanical modifications occur is called the Excavation Damaged Zone (EDZ). The understanding and the quantification of the processes occurring in that zone require accurate experimental tests coupled with advanced numerical modelling. In particular, experimental tests must be designed in order to observe and quantify the damage and the related deformations induced by the gallery excavation. To this end, a non-conventional hollow cylinder test on Boom Clay has been carried out at the LMR-EPFL laboratory to reproduce conditions fairly similar to those that will be experienced by host rocks around disposal galleries. Medium resolution X-Ray Computed Tomography (XRCT) scans have allowed to follow the displacement undergone by Boom Clay and to provide worthwhile data to perform numerical simulations of the encountered processes. Four main features of the Boom Clay behaviour, that are suspected to govern the response of the massif around an excavated gallery, have been underlined: the strong mechanical irreversibilities, the softening behaviour in the vicinity of the hole, the strain localization and the anisotropic response of Boom Clay. The numerical modelling of such a laboratory test is a good mean to extend the observation from laboratory scale to real scale, considering a possible scale effect. This is the reason why it is of paramount importance to validate the numerical models at the scale of laboratory to be able to predict the expected behaviour at the scale of the gallery.

The cross-anisotropic behaviour of Boom Clay, related to its bedding planes, requires a mechanical model that intrinsically considers the anisotropic response of the soil. This feature has been modelled through an extended hardening Drucker-Prager model including elastic and plastic anisotropic relations. It considers that the material is weaker when the principal stress (which is in this case the orthoradial stress) acts at $45^{\circ}$ with respect to its bedding planes which makes the magnitude of 
plasticity higher in the oblique direction. However, the zone in which significant plasticity occurs extends more in the direction of bedding.

In addition to the cross-anisotropic behaviour, the non-symmetric response is also due to the localization of the deformation. For a proper modelling of the strain localization processes, a regularisation method has been used through a hydro-mechanical second grade model. The results of the numerical simulations clearly underline that some shear bands appear around the excavated hole. The strain localizations obtained in the numerical simulations are in good agreement with the "chevron" fracture patterns observed in the field. The complex pattern of shear bands may evolve during the pore pressure redistribution due to transient hydro-mechanical couplings. The accumulation of the shear strain leads to eye-shaped damage zone because of the preferential orientation of the shear bands in the direction of the bedding plane.

\section{$\underline{\text { 7. Acknowledgements }}$}

This project has been co-funded by the European Commission and performed as part of the sixth EURATOM Framework Programme for nuclear research and training activities (2002-2006) under contract FI6W-CT-2007-036449.

\section{References}

Aifantis EC (1984). On the microstructural origin of certain inelastic models. Journal of Engineering Materials and Technology (ASME) 106: 326-330.

Barnichon JD (1998). Finite element modelling in structural and petroleum geology. PhD Thesis, Université de Liège

Barnichon JD, Volckaert G (2003). Observations and predictions of hydromechanical coupling effects in the Boom Clay, Mol Underground Research Laboratory, Belgium. Hydrogeology Journal 2003, 11: 193-202. 
Bastiaens W, Bernier F, Li XL (2006). An overview of long-term HM measurements around HADES URF. EUROCK 2006 Multiphysics Coupling and Long Term Behaviour in Rock Mechanics: $15-26$.

Bazant ZP, Belytschko TB, Chang TP (1984). Continuum model for strain softening. Journal of Engineering Mechanics (ASCE) 110:1666-1692.

Bernier F, Li XL, Bastiaens W (2007). Twenty-five years' geotechnical observation and testing in the Tertiary Boom clay formation. Géotechnique, 57(2): 229-237.

Blümling P, Bernier F, Lebon P, Martin D (2007). The excavation damaged zone in clay formations: time-dependent behaviour and influence on performance assessment. Physics and Chemistry of the Earth, 32: 588-599.

Chambon R, Caillerie D, El Hassan N (1998). One-dimensional localization studied with a second grade model. European Journal of Mechanics - A/Solids 17:637- 656.

Chambon R, Caillerie D, Matsushima T. (2001). Plastic continuum with microstructure, local second gradient theories for geomaterials: localization studies. International Journal of Solids and Structures 38: 8503-8527.

Chapman NA, McKinley IG (1987). The geological disposal of nuclear waste. John Wiley and Sons.

Charlier R (1987). Approche unifiée de quelques problèmes non linéaires de mécanique des milieux continus par la méthode des éléments finis. PhD Thesis, Université de Liège.

Charlier R, Radu JP, Collin F (2001). Numerical modelling of coupled transient phenomena. Revue Française de Génie Civil, 5(6): 719-741.

Chen GJ, Sillen X, Verstricht J, Li XL (2011). ATLAS III in situ heating test in boom clay: Field data, observation and interpretation. Computers and Geotechnics 38(5): 683-696.

Collin F (2003). Couplages thermo-hydro-mécaniques dans les sols et les roches tendres partiellement saturés. PhD Thesis, Université de Liège.

Collin F, Chambon R, Charlier R (2006). A finite element method for poro mechanical modelling of geotechnical problems using local second gradient models. International Journal of Numerical Methods in Engineering, 65: 1749-1772. 
Collin F, Li XL, Radu JP, Charlier R (2002). Thermo-hydro-mechanical coupling in clay barriers. Engineering Geology, 64: 179-193.

Cosserat E, Cosserat F (1909). Théorie des corps déformables. A Hermann et Fils: Paris.

Davies C, Bernier F (2005). Impact of the excavation disturbed or damaged zone (EDZ) on the performance of radioactive waste geological repositories. Proc. of the European Commission Cluster. Conference and Workshop, Luxembourg.

de Borst R, Muhlhaus H (1992). Gradient dependant plasticity: formulation and algorithmic aspects. International Journal for Numerical Methods in Engineering 35: 521-539.

Desrues J, Viggiani G (2004). Strain localization in sand: an overview of the experimental results obtained in Grenoble using stereophotogrammetry. International Journal for Numerical and Analytical Methods in Geomechanics 2004;, 28(4):279 -321.

Dizier A (2011). Caractérisation des effets de températures dans la zone endommagée autour de tunnel de stockage de déchets nucléaires dans des roches argileuses. Phd thesis, Université de Liège, Belgium.

Drucker DC, Prager W (1952). Solid mechanics and plastic analysis for limit design. Quarterly of Applied Mathematics, 10(2): 157-165.

Duveau G, Shao JF, Henry JP (1998). Assessment of some failure criteria for strongly anisotropic geomaterials. Mechanics of cohesive-frictional materials, 3, 1-26.

Ehlers W, Volk W (1998). On theoretical and numerical methods in the theory of porous media based on polar and non-polar elasto-plastic solid materials. International Journal of Solids and Structures 35: 4597- 4617.

François B, Collin F, Dizier A, Charlier R (2011). Development of an extended Drucker-Prager hardening model for cross-anisotropy of soft rocks. 2nd International Symposium on Computational Geomechanics (ComGeo II), Cavtat-Dubrovnik, Croatia, 142-152.

François B, Laloui L, Laurent C (2009). Thermo-hydro-mechanical simulation of ATLAS in-situ large scale test in Boom Clay. Computer and Geotechnics, 36: 626-640. 
Gens A, Olivella S (2001). Clay barrier in radioactive waste disposal. Revue Française de Génie Civil, 5(6): 845-856.

Germain P (1973a). La méthode des puissances virtuelles en mécanique des milieux continus: Première partie: théorie du second gradient. Journal de Mécanique 12(2):235 -274.

Germain P (1973b). The method of virtual power in continuum mechanics part 2: microstructure. SIAM Journal on Applied Mathematics 25(3):556 -575.

Graham J, Houlsby GT (1982) Anisotropic elasticity of a natural clay. Géotechnique 33(2), 165-180.

Labiouse V, Vietor T (2013). Laboratory and in situ simulation tests of the Excavation Damaged Zone around galleries in Opalinus Clay. Rock Mechanics and Rock Engineering. This issue.

Labiouse V, Sauthier C, You S (2013). Hollow cylinder simulation experiments of galleries in Boom Clay formation. Rock Mechanics and Rock Engineering. This issue.

Laloui L, François B, Nuth M, Peron H, Koliji A (2008). A thermo-hydro-mechanical stress-strain framework for modelling the performance of clay barriers in deep geological repositories for radioactive waste. 1st European Conf. on Unsaturated Soils, Durham, United Kingdom: 6380.

Lekhnitskii SG (1977). Theory of elasticity of an anisotropic elastic body. 2nd Edition, Moscow, MIR Publishers.

Matsushima T, Chambon R, Caillerie D (2002). Large strain finite element analysis of a local second gradient model : application to localization. International Journal for Numerical Methods in Engineering, 54(4):499-521.

Mindlin R (1965) Second gradient of strain and surface-tension in linear elasticity. International Journal of Solids and Structures 1:417- 438.

Mindlin RD, Eshel NN (1968). On first strain gradient theories in linear elasticity. International Journal of Solids and Structures 4:109-124.

Muhlhaus H, Vardoulakis I (1987). The thickness of shear bands in granular materials. Géotechnique 37: 271-283. 
Papamichos E, van den Hoek P (1995). Size dependency of Castlegate and Berea sandstone hollowcylinder strength on the basis of bifurcation theory, In: Proc. 35th US Symp. Rock Mechanics, 1995, 301-306.

Papanastasiou P, Vardoulakis I, (1989) Bifurcation analysis of deep boreholes: II. Scale effect, International Journal for Numerical and Analytical Methods in Geomechanics, 13(2), 183-198

TIMODAZ (2010). Timodaz Report - Deliverable D5 - THM characterisation and input for simulations. Editor: P. Delage, Commission of the European Communities. -

Tsang CF, Bernier F, Davies C (2005). Geohydromechanical processes in the excavation damaged zone in crystalline rock, rock salt, and indurated and plastic clays in the context of radioactive waste disposal. Int. J. Rock Mech. Min. Sci., 42(1): 109-125.

Vardoulakis I, Sulem J (1995). Bifurcation Analysis in Geomechanics. Blackie Academic and Professional: London.

Zervos A, Papanastasiou P, Vardoulakis I (2001). Modelling of localisation and scale effect in thickwalled cylinders with gradient elastoplasticity. Int. J. Solids Structures, 38:5081-5095.

Zervos A, Papanastasiou P, Vardoulakis I (2008) Shear localisation in thick-walled cylinders under internal pressure based on Gradient Elastoplasticity. Journal of Theoretical and Applied Mechanics, 38 81-100 


\begin{tabular}{lll}
\hline Young modulus [MPa] & $\mathrm{E}$ & 300 \\
Poisson ratio [-] & $\mathrm{v}$ & 0.125 \\
Friction angle [ $^{\circ}$ ] & $\phi^{\prime}$ & 18 \\
Cohesion [MPa] $_{\left.\text {Dilatation angle [ }{ }^{\circ}\right]}$ & $\mathrm{c}^{\prime}$ & 0.3 \\
Intrinsic saturated permeability $\left[\mathrm{m}^{2}\right]$ & $\psi$ & $0-10$ \\
Porosity [-] & $\mathrm{k}_{\mathrm{w}}$ & $2-4 \times 10^{-19}$ \\
\hline
\end{tabular}

Table 1: Drained geomechanical characteristics of Boom Clay (Bernier et al. 2007) 


\begin{tabular}{|c|c|c|c|}
\hline \multirow{2}{*}{\multicolumn{2}{|c|}{ Geomechanical characteristics }} & \multicolumn{2}{|c|}{ Drucker-Prager } \\
\hline & & Isotropic & Anisotropic \\
\hline Young elastic modulus [MPa] & $E_{/ /}$ & 300 & 400 \\
\hline Young elastic modulus [MPa] & $E_{\perp}$ & 300 & 200 \\
\hline Poisson ratio [-] & $v_{\text {IIIII }}$ & 0.125 & 0.125 \\
\hline Poisson ratio [-] & $v_{/ / 1}$ & 0.125 & 0.125 \\
\hline Shear modulus [MPa] & $G_{/ / \perp}$ & $133^{*}$ & $\begin{array}{l}178 \\
255\left(0^{\circ}\right.\end{array}$ \\
\hline Initial cohesion $[\mathrm{kPa}]$ & $c^{\prime}{ }_{i}$ & 300 & $\begin{array}{l}240\left(45^{\circ}\right) \\
330\left(90^{\circ}\right)\end{array}$ \\
\hline Final cohesion [kPa] & $c_{f}^{\prime}$ & 100 & $\begin{array}{l}85\left(0^{\circ}\right) \\
80\left(45^{\circ}\right) \\
110\left(90^{\circ}\right)\end{array}$ \\
\hline Softening parameter [-] & $B_{c}$ & 0.01 & 0.01 \\
\hline $\begin{array}{l}\text { Initial friction angle }\left[^{\circ}\right] \\
\text { Final friction angle }\left[{ }^{\circ}\right]\end{array}$ & $\begin{array}{l}\emptyset_{i}^{\prime} \\
\varnothing_{f}^{\prime}\end{array}$ & $\begin{array}{l}5 \\
18\end{array}$ & $\begin{array}{l}5 \\
18\end{array}$ \\
\hline Hardening parameter [-] & $B_{p}$ & 0.01 & 0.01 \\
\hline Dilatancy angle $\left[{ }^{\circ}\right]$ & $\psi$ & 0 & 0 \\
\hline
\end{tabular}

Table 2: Set of the Boom Clay geomechanical parameters used with the isotropic and anisotropic Drucker-Prager model. 


\begin{tabular}{|c|c|c|}
\hline Hydraulic characteristics & & \\
\hline Initial porosity & $n_{0}$ & 0.39 \\
\hline Intrinsic permeability [m²] & $k_{w}$ & $4.10^{-19}$ \\
\hline $\begin{array}{l}\text { Water specific mass }\left[\mathrm{kg} / \mathrm{m}^{3}\right] \\
\text { Fluid dynamic viscosity [Pa.s] }\end{array}$ & $\begin{array}{l}\rho_{w} \\
\mu_{w}\end{array}$ & $\begin{array}{l}1000 \\
10^{-3}\end{array}$ \\
\hline
\end{tabular}

Table 3: Set of the Boom Clay hydraulic parameters. 


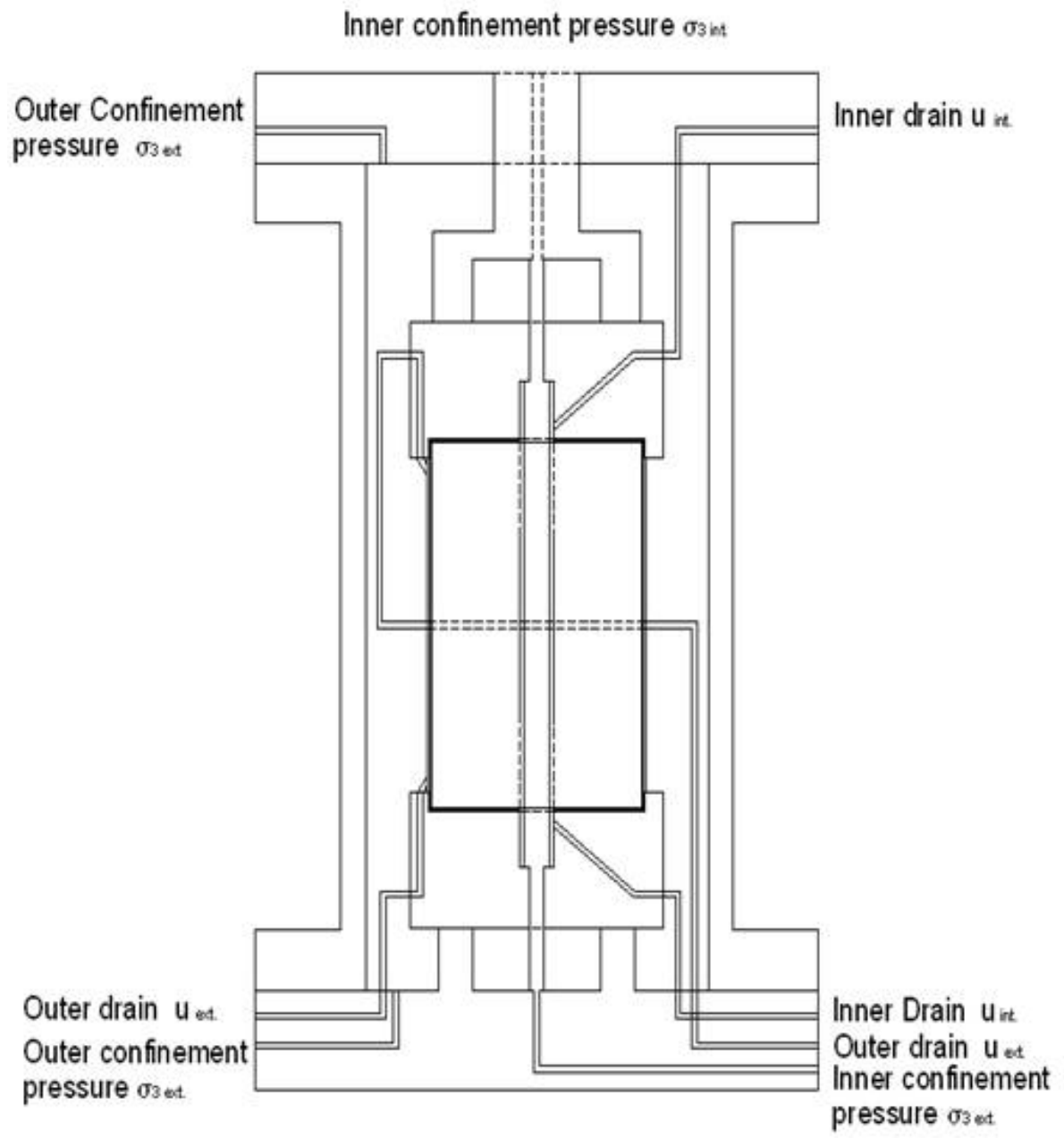

Figure 1: Sketch of the hollow cylinder device. 

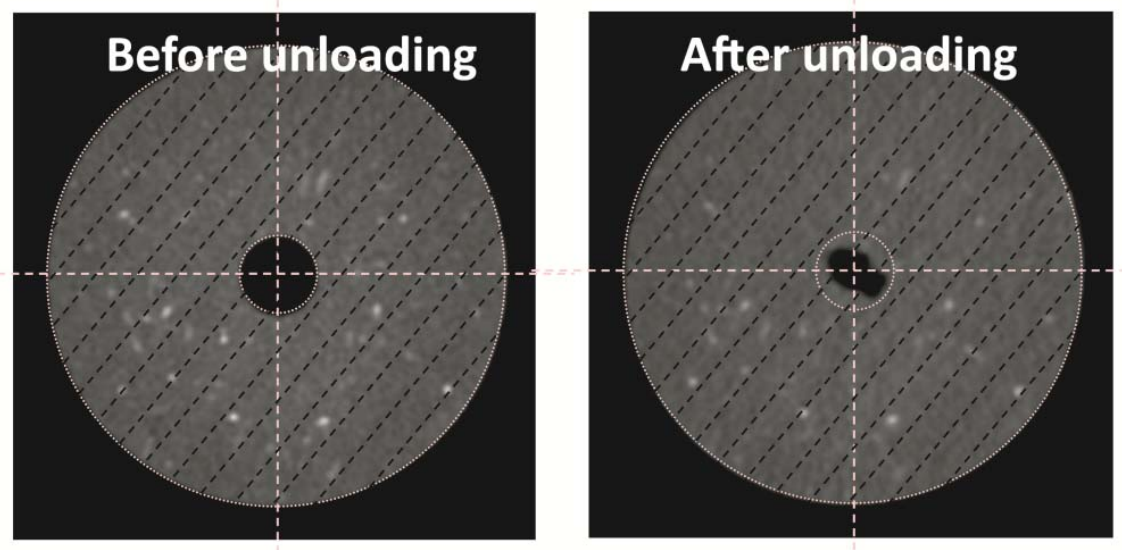

Figure 2: Deformed cross-section observed by the X-Ray tomography scan and compared with the initial section. The direction of the bedding plane has been represented by the dashed lines (Labiouse et al. 2013). 


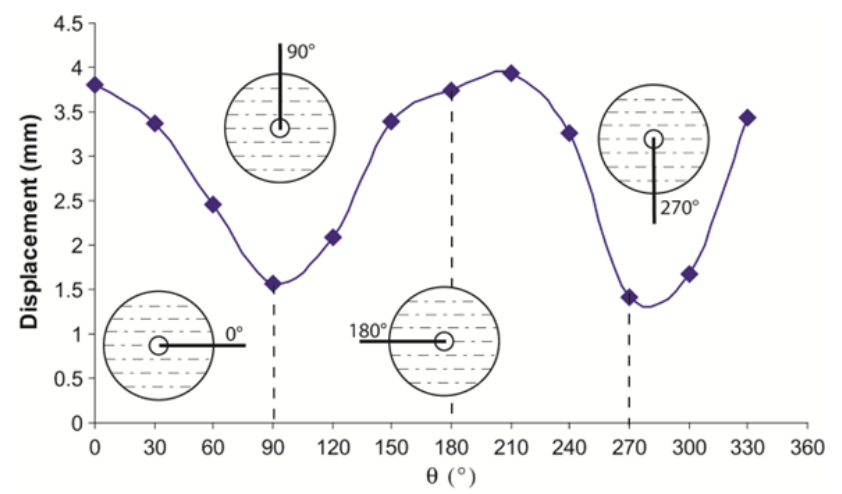

Figure 3: Experimental measurements of the central hole displacement at the end of the mechanical unloading and pore water pressure stabilisation. Orientations $0^{\circ}$ and $180^{\circ}$ refer to the direction parallel to bedding. 


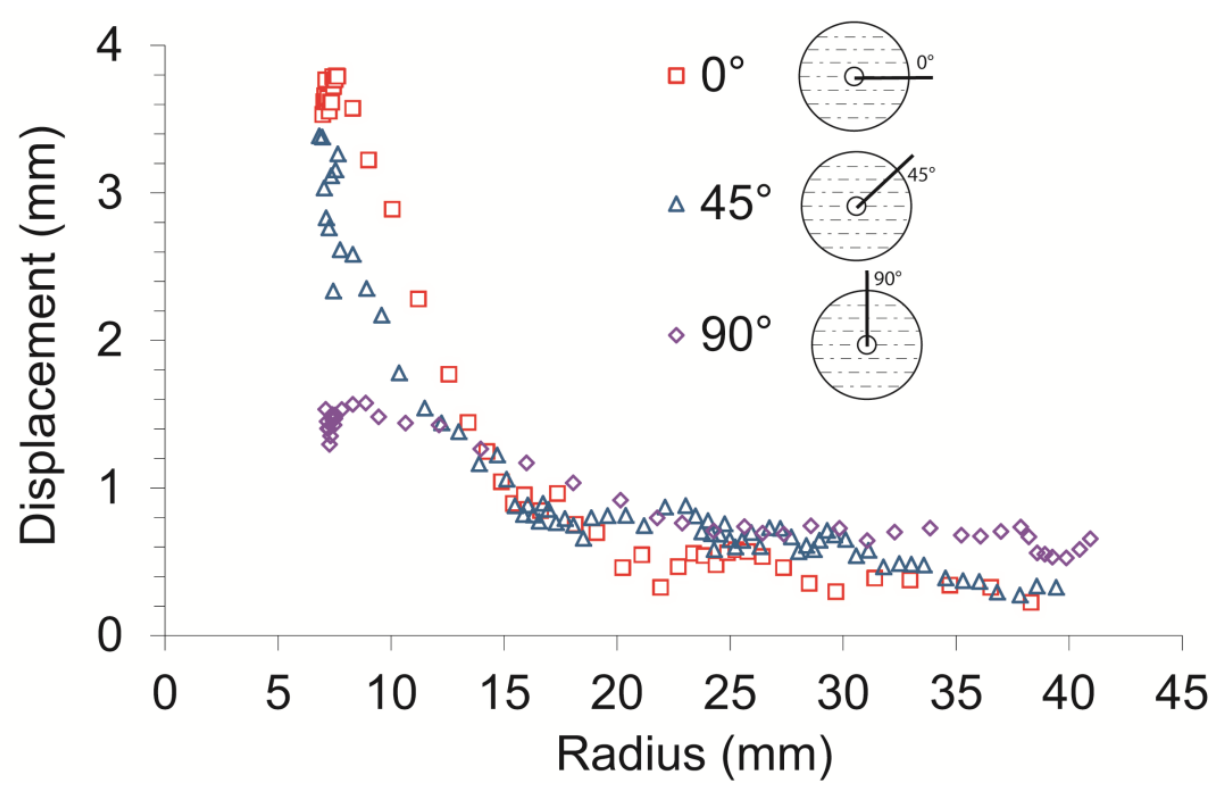

Figure 4: Displacement profile in three different radial directions reported as a function of the distance from the centre of the hole: parallel to bedding plane $\left(0^{\circ}\right)$ at $45^{\circ}$ and perpendicular to bedding $\left(90^{\circ}\right)$. 
a)

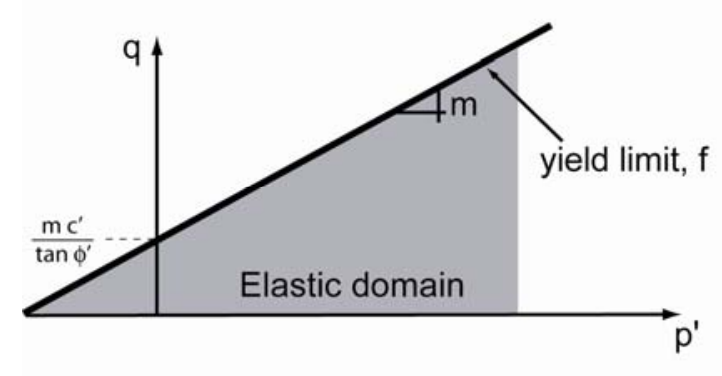

b)

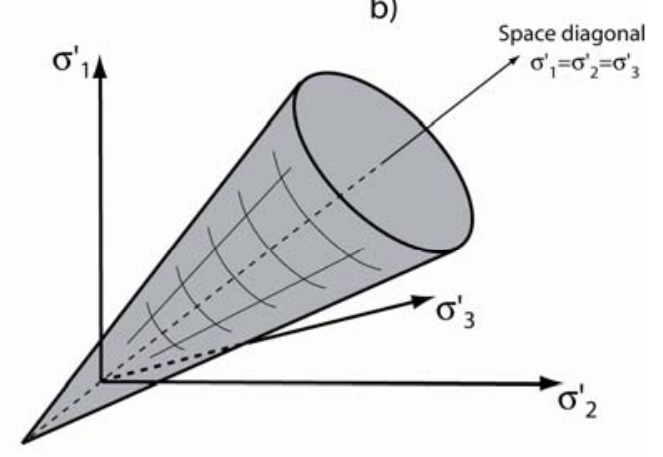

Figure 5: Yield limit of the model. (a) View in the ( $\left.p^{\prime}-q\right)$ plane and (b) in the principal stress space. 


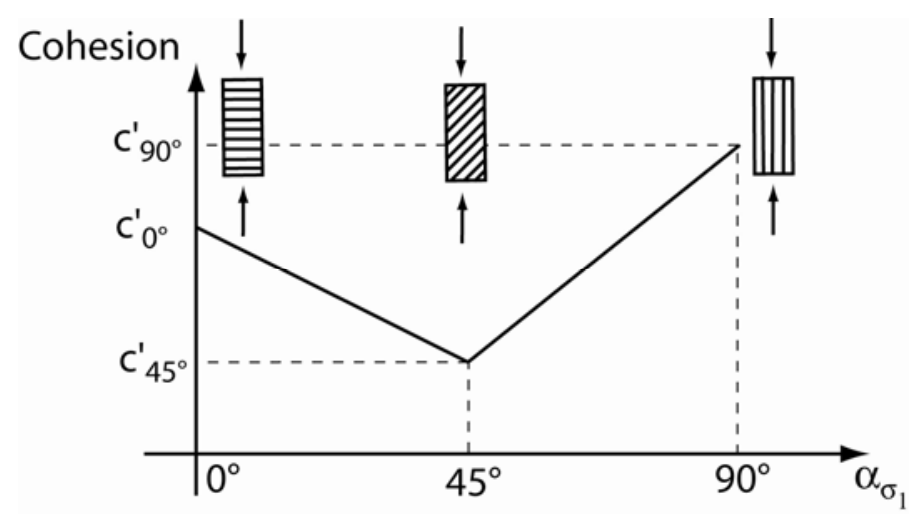

Figure 6: Schematic view of the cohesion evolution as a function of the angle between the normal vector to bedding plane and the direction of major principal stress. 


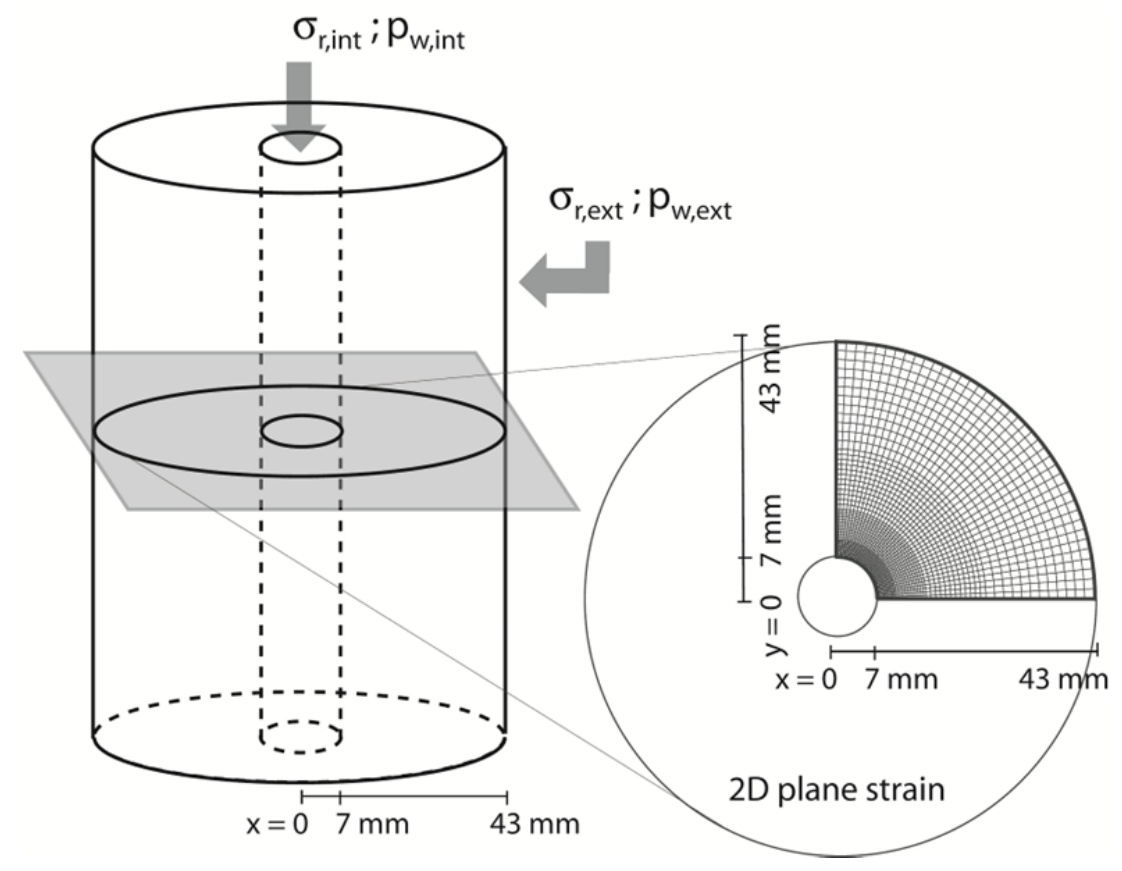

Figure 7: Modelling of the hollow cylinder under two-dimensional plane strain conditions. 


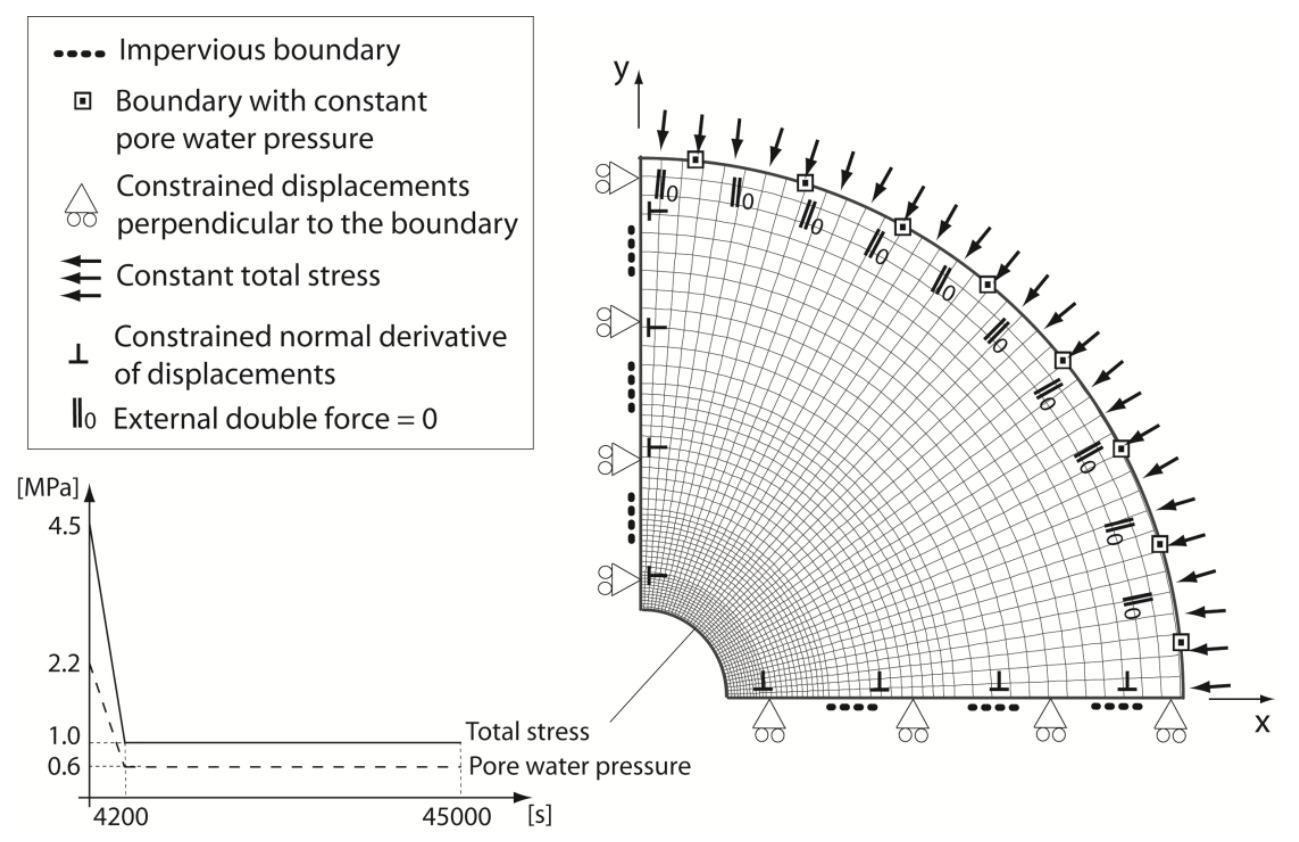

Figure 8: Finite element mesh and hydro-mechanical boundary conditions of the 2D plane strain problem, including additional boundary conditions for second gradient model. 


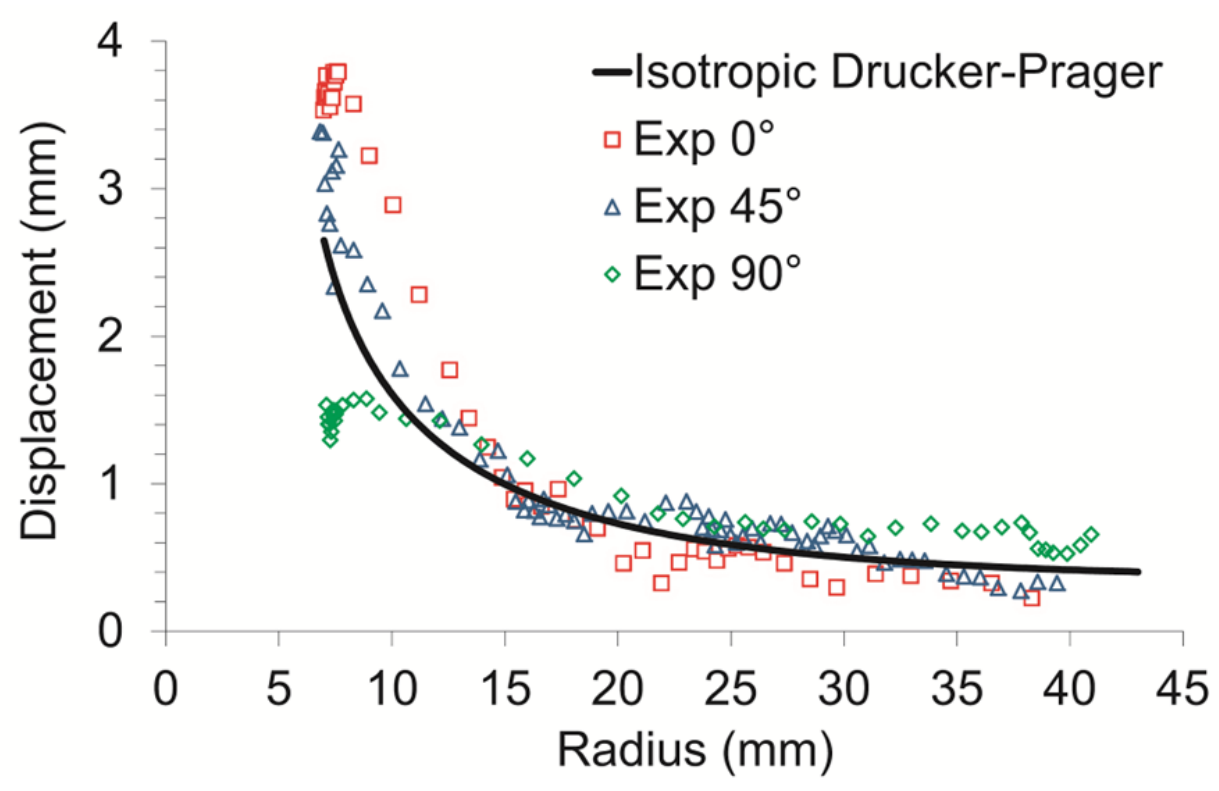

Figure 9: Radial profile of displacements. Comparison between experimental measurements in three different directions and numerical results obtained with the isotropic Drucker-Prager model. 
a)

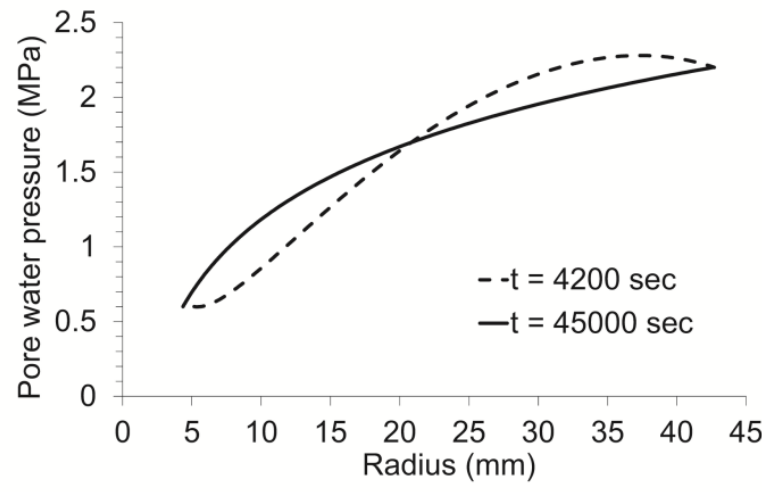

c)

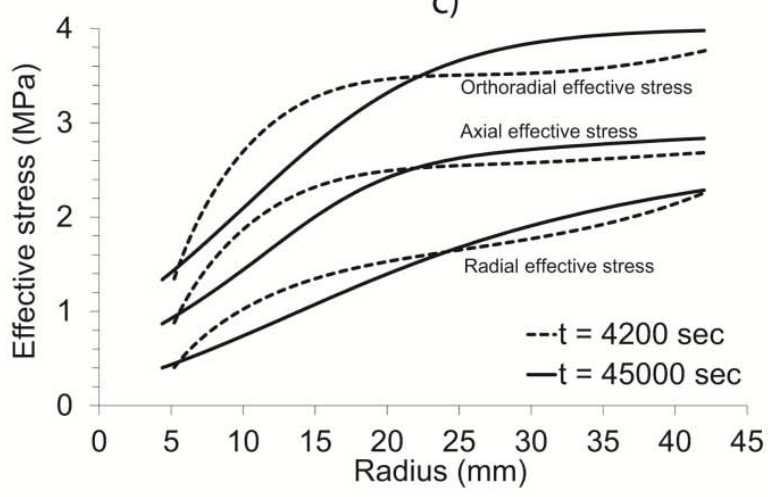

b)

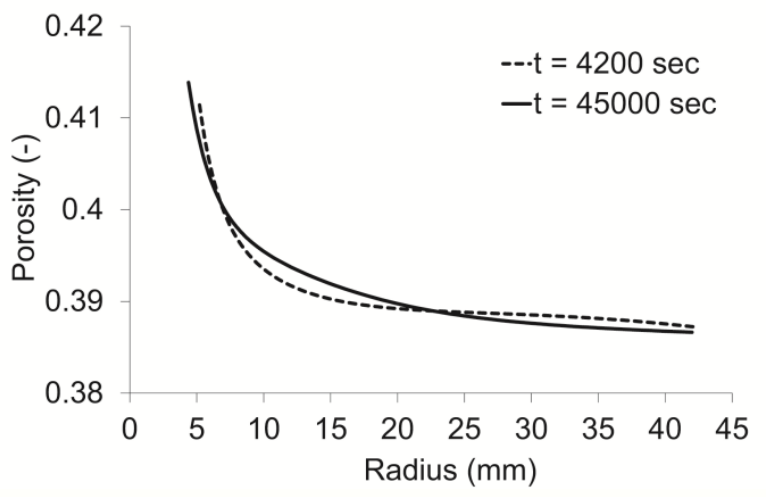

d)

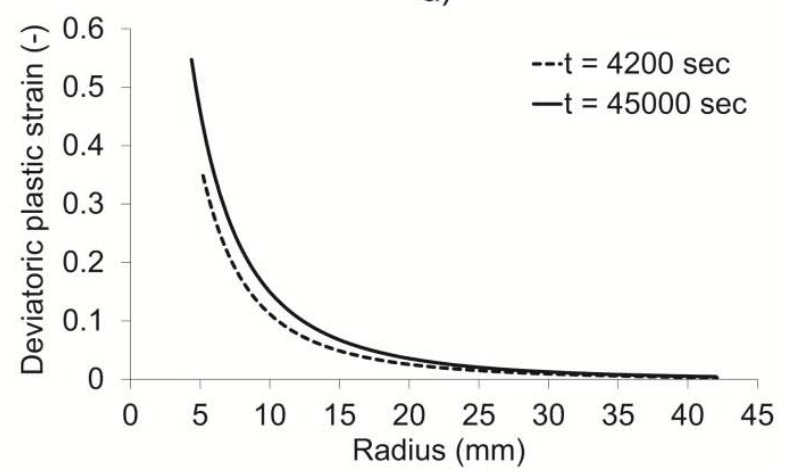

Figure 10: Prediction of the isotropic Drucker-Prager model: Radial profile just after the mechanical unloading ( $t=4200 \mathrm{~s})$ and at the end of the pore water pressure dissipation $(t=45000$ s): (a) the pore water pressure, (b) the porosity, (c) the three principal stresses, (d) the deviatoric plastic strain. 


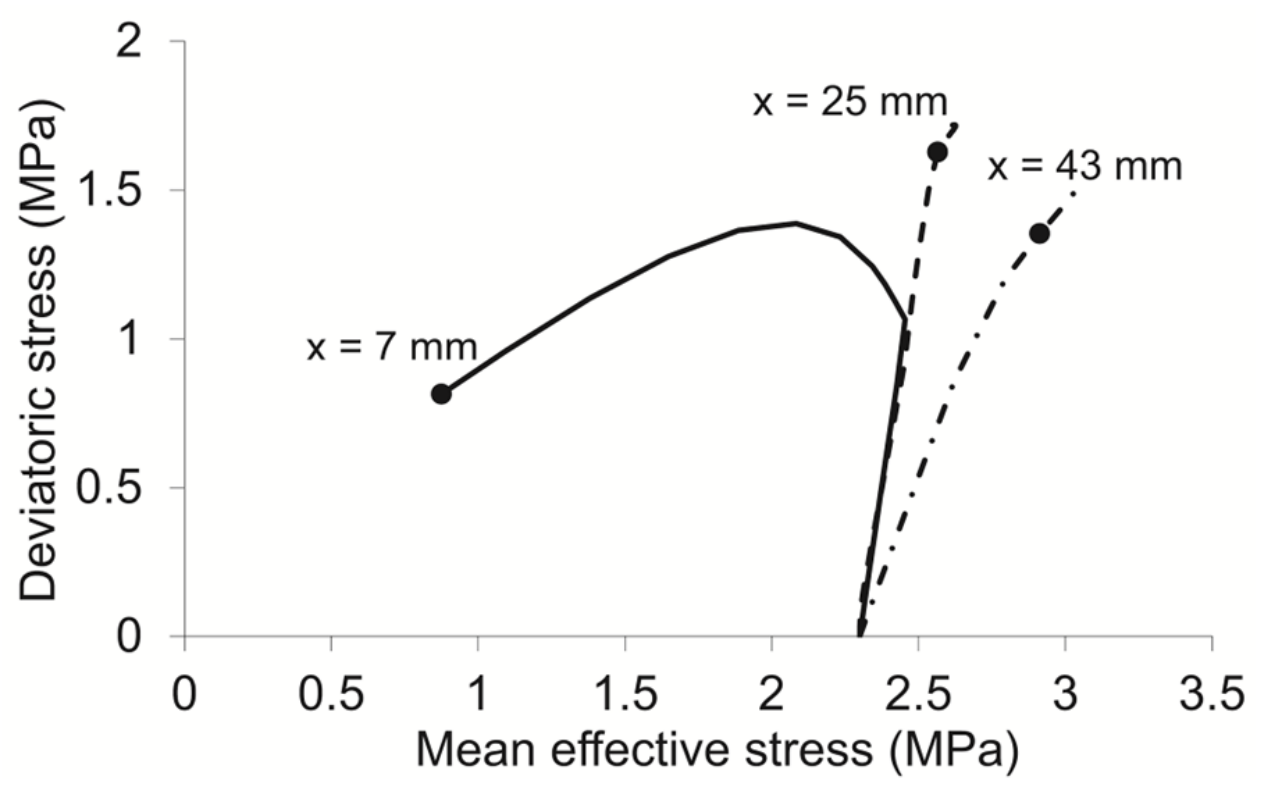

Figure 11: Predicted stress paths in the (p'-q) plane at three different radial coordinates $(7 \mathrm{~mm}$, $25 \mathrm{~mm}$ and $43 \mathrm{~mm}$ ) using the isotropic Drucker-Prager model. The points represent the end of the mechanical unloading $(t=4200 \mathrm{~s})$ 


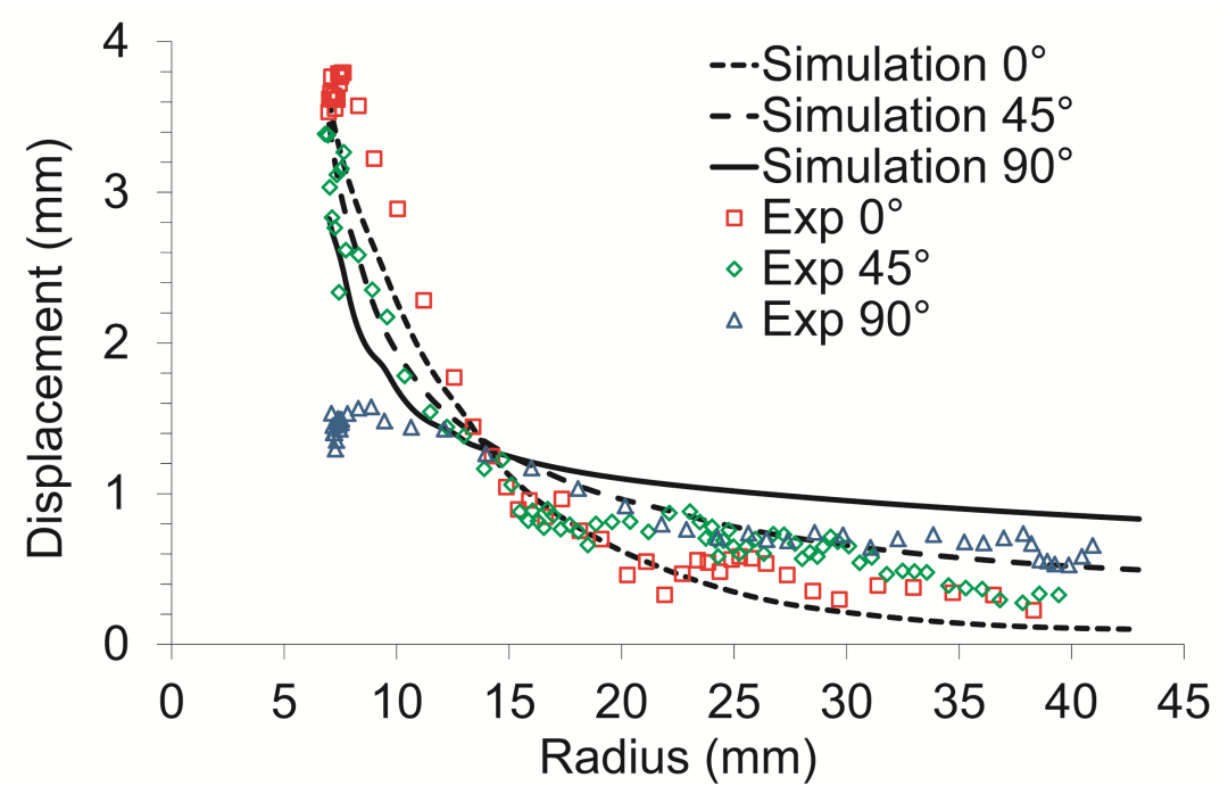

Figure 12: Radial profile of displacements. Comparison between experimental measurements in three different directions and numerical results obtained with the anisotropic Drucker-Prager model. 

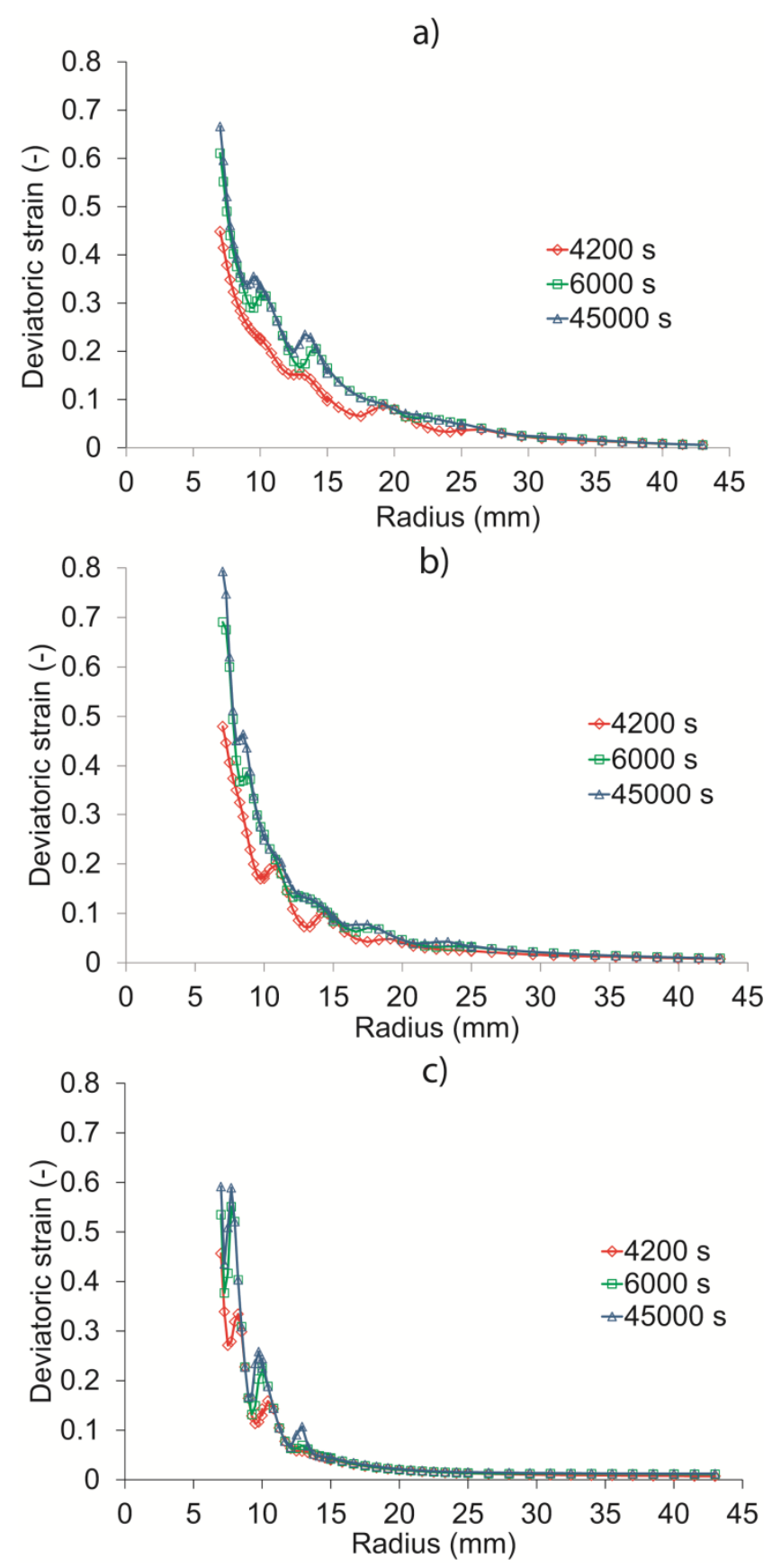

Figure 13: Radial profile of the deviatoric strain along the (a) horizontal $\left(\theta=0^{\circ}\right)$, (b) oblique $(\theta=$ $\left.45^{\circ}\right)$ and (c) vertical $\left(\theta=90^{\circ}\right)$ directions, obtained with the cross-anisotropic Drucker-Prager model. 


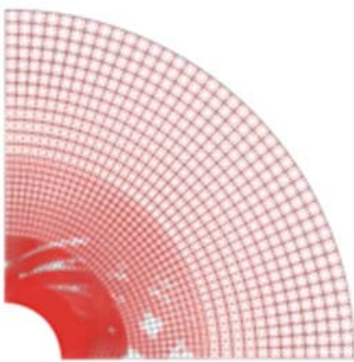

a)

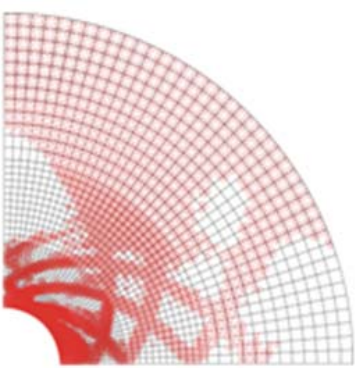

c)

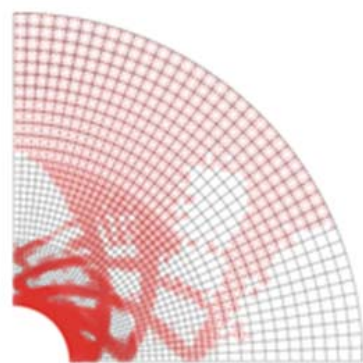

e)

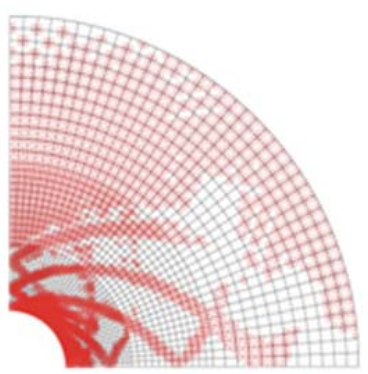

g)

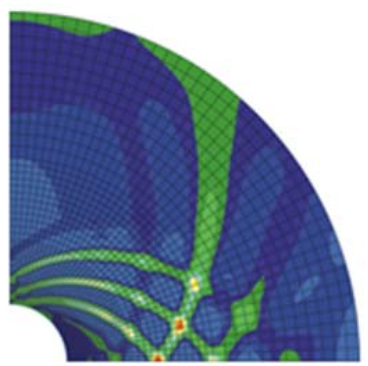

b)

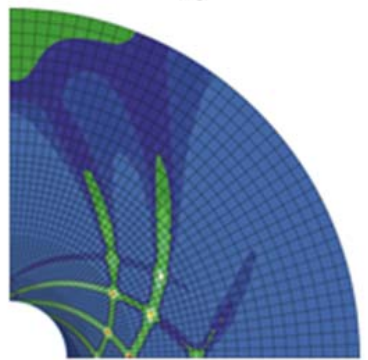

d)

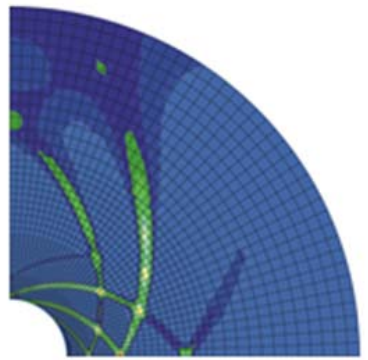

f)

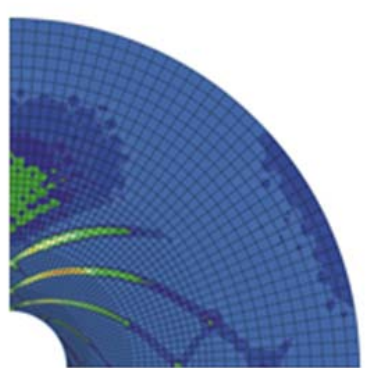

h)

Figure 14: Plastic point map (left) and contour of normalized increment of deviatoric strain (right) at $\mathrm{t}=4200 \mathrm{~s}(\mathrm{a}-\mathrm{b}), \mathrm{t}=5000 \mathrm{~s}(\mathrm{c}-\mathrm{d}), \mathrm{t}=6000 \mathrm{~s}(\mathrm{e}-\mathrm{f})$ and $\mathrm{t}=10000 \mathrm{~s}(\mathrm{~g}-\mathrm{h})$ obtained with the cross-anisotropic Drucker-Prager model. 
(a)

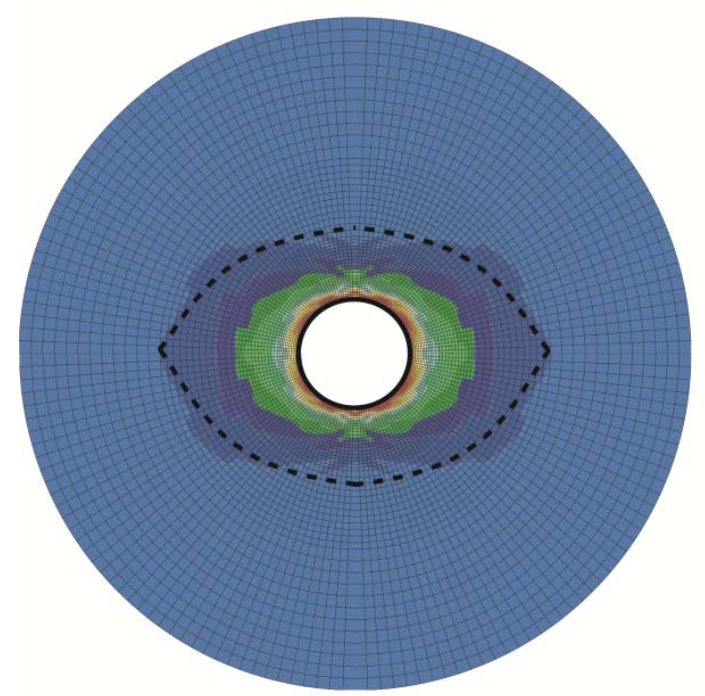

(b)

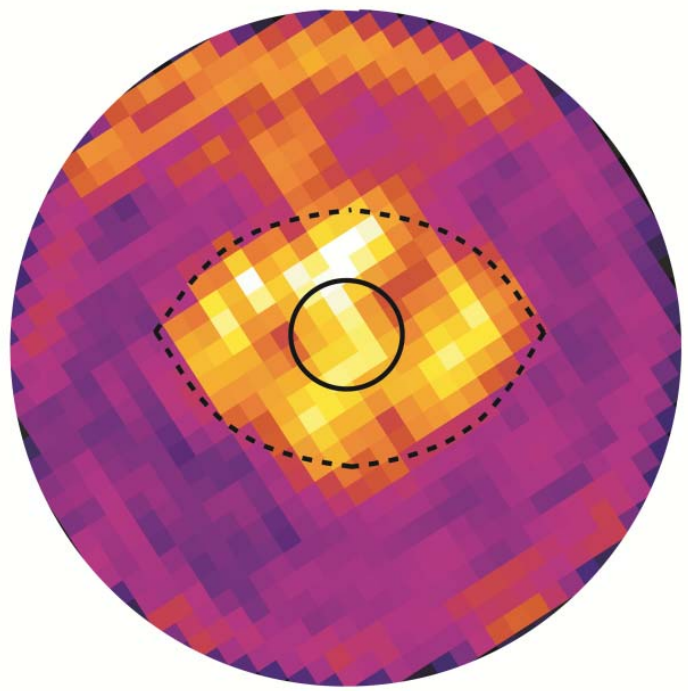

Figure 15: Qualitative comparison of the shape of the excavation damaged zone between numerical prediction (a) and image correlation obtained from X-Ray tomography scan (b). The pictures show the cumulative deviatoric strain at the end of the test. Dashed line has been added to reveal the eye-shaped damaged zone. 